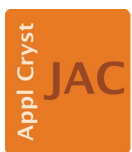

JOURNAL OF APPLIED CRYSTALLOGRAPHY

ISSN 1600-5767

Received 11 June 2020

Accepted 18 December 2020

Edited by S. Boutet, SLAC National Accelerator Laboratory, Menlo Park, USA

Keywords: X-ray optics; bent Laue crystal diffraction; ray tracing; energy-dispersive $\mathrm{X}$-ray imaging.

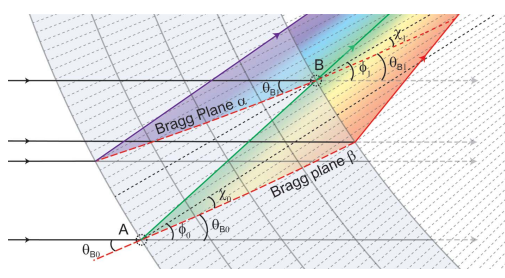

C 2021 International Union of Crystallography

\section{Bent Laue crystal anatomy: new insights into focusing and energy-dispersion properties}

\author{
Peng Qi, ${ }^{a *}$ Xianbo Shi, ${ }^{b}$ Nazanin Samadi ${ }^{\mathrm{c}}$ and Dean Chapman ${ }^{\mathrm{d}}$ \\ a'Division of Biomedical Engineering, University of Saskatchewan, Saskatoon, Canada, ${ }^{\mathbf{b}}$ Advanced Photon Source, \\ Argonne National Laboratory, Lemont, Illinois, USA, 'Swiss Light Source, Paul Scherrer Institute, 5232 Villigen, \\ Switzerland, and ${ }^{\mathbf{d} A n a t o m y, ~ P h y s i o l o g y ~ a n d ~ P h a r m a c o l o g y, ~ U n i v e r s i t y ~ o f ~ S a s k a t c h e w a n, ~ S a s k a t o o n, ~ C a n a d a . ~}$ \\ *Correspondence e-mail: peng.qi@usask.ca
}

X-ray Laue-type monochromators are common and essential optical components at many high-power X-ray facilities, e.g. synchrotron facilities. The X-ray optics of bent Laue crystals is a well developed area. An incident X-ray beam penetrating a bent Laue crystal will result in a diffracted beam with different angles and energies. There is a need for a way of organizing the rays that allows one to sort out the energy and spatial properties of the diffracted beam. The present work introduces a new approach for describing the general behaviour of bent Laue crystals from a ray-tracing point of view. This quasi-monochromatic beam approach provides an intuitive view of bent-crystal diffraction and leads to deeper understanding. It explains the energy and spatial properties of common and special cases of bent Laue optics, predicts phenomena that can improve energy-dispersion-related X-ray imaging techniques and provides a theoretical framework that makes ray-tracing simulation easier to realize.

\section{Introduction}

X-ray Laue- or transmission-type monochromators have been used for many types of application, especially in high-X-rayenergy applications where the Laue geometry is better at handling the high incident powers that are generated by synchrotron or other high-power X-ray sources. The description of bent Laue crystal optics is a well developed area. The difficulty in understanding the behaviour of a bent Laue crystal is that a single incoming polychromatic ray gives rise to a multiplicity of diffracted beams, all with different angles and energies. The situation is further complicated when several incoming beams intercept the crystal. Although the energy and trajectory of these rays are predictable, understanding the focusing and energy-dispersive properties of the diffracted beams is hindered by having to account for all of these rays. What is needed is a way of organizing the rays that allows one to sort out the energy and spatial properties of the diffracted beam.

In the present work, a general description of bent Laue optics is introduced that (i) explains the path of diffracted $\mathrm{X}$-rays, the focusing properties and the energy properties in simple geometric terms, and (ii) predicts a new phenomenon of monochromatic focusing which may enable new bent Laue energy-dispersive applications or improvement of existing ones.

This new approach is best understood from the 'magic condition' (Zhu et al., 2014; Martinson et al., 2015; Qi et al., $2019 b$ ) that allows bent Laue crystals to provide a small focal 
size and good energy resolution simultaneously, as described in the next section.

\section{Background}

Often, elastic bending of crystals is desired to focus or increase the energy dispersion of the diffracted X-ray beams. The diffraction properties of elastically bent Laue crystals can be described by wave theories (Takagi, 1969; Taupin, 1964; Penning \& Polder, 1961) and lamella models (Suortti et al., 1994). For the discussion that follows, the crystals will be elastically bent single crystals such as silicon.

Earlier work (Schulze \& Lienert, 1996; Schulze et al., 1998) has shown that the focal size of an asymmetric bent Laue monochromator can be minimized by coinciding the geometric focus and the single-ray focus (Fig. 1) with a carefully chosen asymmetry angle. Subsequent studies reported that at this condition the angle-energy dispersion of the diffracted polychromatic beam is improved in addition to focal-size minimization (Zhu et al., 2014). Novel imaging techniques (Zhu et al., 2014; Martinson et al., 2015; Bassey et al., 2016; Qi, Samadi et al., 2019) have been developed to take advantage of this 'magic condition'.

However, the mechanism of the energy properties of the magic condition is unclear. The coinciding of the geometric focus and single-ray focus guides the way to the magic condition but leaves its optical attributes unknown. The present work unveils the magic condition optics from the perspective of diffraction angles at representative locations in the crystal and provides a new way of studying bent Laue monochromators which makes ray-tracing easier to realize.

In this work, we assume a crystal is singly bent around an axis perpendicular to the diffraction plane, and because we are looking at diffraction from the ray-tracing point of view, we assume that each diffracted ray is not diffracted again.
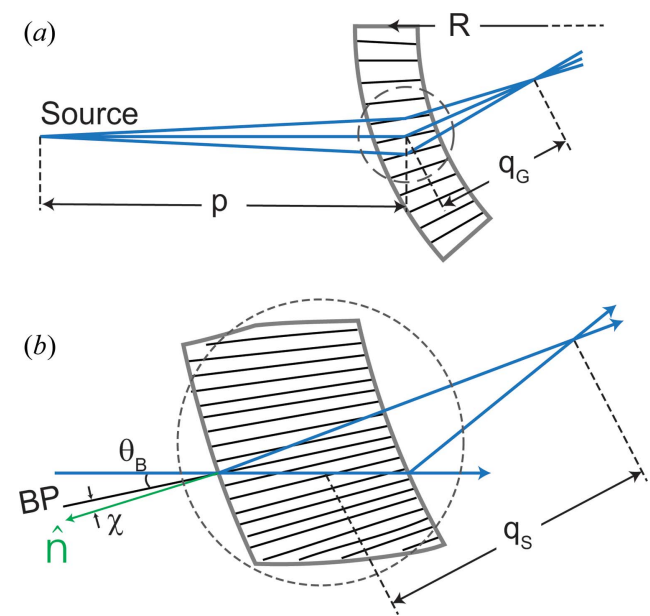

Figure 1

Bent asymmetric Laue focusing. (a) Geometric focusing with the source at a distance $p$, a bent Laue crystal of radius $R$, asymmetry angle $\chi$, Bragg angle $\theta_{\mathrm{B}}$ and focal distance $q_{\mathrm{G}}$. $(b)$ A chosen relationship between the incident ray in relation to the Bragg plane (BP), the crystal surface normal $(\hat{n})$ and the asymmetry angle $\chi$. The geometry shown results in the single-ray focus at a distance $q_{\mathrm{S}}$.

\section{Magic condition}

3.1. Concept

As demonstrated in Fig. 2, the coincident state of the geometric focus and the single-ray focus is directly related to the equality of the Bragg angles $\theta_{\mathrm{B}}$ at points $A$ and $B$ because of Bragg's law, where $B$ is the location where the diffracted X-rays from point $A$ exit the crystal. Assuming the incident $\mathrm{X}$-ray beam is parallel, when the two Bragg angles are equal to each other, the travel paths of the X-rays diffracted from $A$ and $B$ coincide, which makes the geometric focus and the single-ray focus coincide in consequence. Therefore, the determining condition can be transformed from matching the two foci to equating the two Bragg angles of the diffracted rays from the points $A$ and $B$. A detailed microscopic view is shown in Fig. 3. The $\beta$ plane can be considered as an extension of the $\alpha$ plane by translating and 'stitching' the $\beta$ plane so that point $A$ is coincident with point $B$ at the end of the $\alpha$ plane. When the equal Bragg angle condition is met, the planes $\alpha$ and $\beta$ behave like they are stitched together and form an extended Bragg plane. The diffracted X-rays from the two planes become continuously energy dispersive as if diffracted from one plane. The diffraction angles are the same not only at points $A$ and $B$ but at every point on the path from $A$ to $B$. Mathematically, this implies that the magic condition is thickness independent [as indicated by equation (12) in Section 3.3].

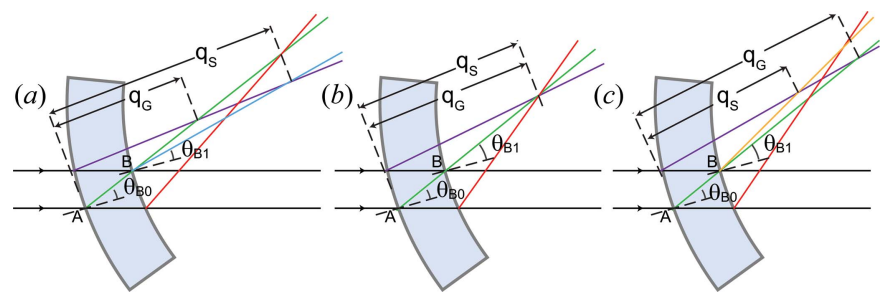

Figure 2

Geometric focus $q_{\mathrm{G}}$, single-ray focus $q_{\mathrm{S}}$ and Bragg angles when the magic condition is met or not met. (a) The geometric focus is less than the single-ray focus and the Bragg angle at $B$ is smaller than that at $A$. (b) The geometric focus equals the single-ray focus and the Bragg angle at $B$ equals that at $A$ (the magic condition). (c) The geometric focus is greater than the single-ray focus and the Bragg angle at $B$ is larger than that at $A$.

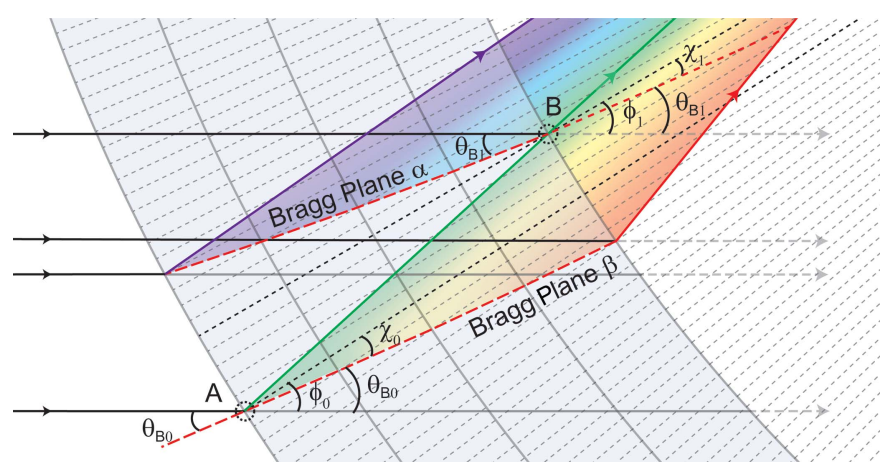

Figure 3

Magic condition concept geometry for an infinitely distant source. 


\subsection{Magic condition with infinite source distance}

When the magic condition is met, X-rays diffracted at points $A$ and $B$ travel on the same path. This can be directly translated to $\theta_{\mathrm{B}_{0}}=\theta_{\mathrm{B}_{1}}$, where the subscripts 0 and 1 correlate to points $A$ and $B$, respectively, as shown in Fig. 3. In general, the diffraction angle $\theta_{\mathrm{B}}$ can be described by $\chi$ and $\phi$,

$$
\theta_{\mathrm{B}}=\phi-\chi,
$$

where $\phi$ is the directional angle from the X-ray incident direction to the crystal surface normal (short-dashed lines in Fig. 3) and $\chi$ (the asymmetry angle) is the directional angle from the diffraction lattice (red long-dashed lines in Fig. 3, such as the $\alpha$ and $\beta$ planes) to the surface normal. (The angle $\phi$ is not a direct parameter for describing the crystal but is helpful for simplifying the discussion hereinafter.) The magic condition can then be described with the changes in $\chi$ and $\phi$ summing to zero,

$$
\theta_{\mathrm{B}_{1}}-\theta_{\mathrm{B}_{0}}=\left(\phi_{1}-\phi_{0}\right)-\left(\chi_{1}-\chi_{0}\right)=\Delta \phi-\Delta \chi=0 .
$$

The change in $\chi$ is caused by the deformation of the bent crystal. As shown in Fig. 3, the concave (convex) side of the crystal is compressed (expanded) in one dimension along the crystal surface but perpendicular to the bending axis, which results in elastic expansion (compression) in the two transverse dimensions. The change in $\chi$ is given by

$$
\Delta \chi=-\frac{1+v}{2} \frac{T}{R} \sin 2 \chi
$$

(see Appendix $A 1$ for a detailed derivation). Here $v$ is the Poisson ratio of the crystal for the given crystal orientation $(\chi$, diffraction indices and bending axis), $R$ is the crystal bending radius and $T$ is the bent-crystal thickness, which is related to the flat-crystal thickness $T_{0}$ by (Qi, Shi et al., 2019)

$$
T=\frac{2 R}{v} \sinh \left(\frac{\nu T_{0}}{2 R}\right) \text {. }
$$

The change in $\phi$ can be determined from the magic condition geometry and is given by

$$
\Delta \phi_{\infty}=-\frac{T}{R} \tan \left(\theta_{\mathrm{B}}-\chi\right),
$$

where the subscript $\infty$ indicates the scenario of a source at infinite distance. $\Delta \phi$ in general will be given in Section 3.3 (See Appendix $A 2$ for a detailed derivation.)

Therefore, the magic condition equation when the source distance is infinite is given by

$$
\Delta \phi_{\infty}-\Delta \chi=\frac{1+v}{2} \frac{T}{R} \sin 2 \chi-\frac{T}{R} \tan \left(\theta_{\mathrm{B}}-\chi\right)=0 .
$$

\subsection{Magic condition with finite source distance}

When the source distance is finite, the diffracted X-rays exiting in the same path to the common focus are diffracted by slightly different Bragg angles. The variation in Bragg angles between points $A$ and $B$ (Fig. 4) is

$$
\Delta \theta_{\mathrm{B}_{01}}=-\frac{\psi}{2}
$$

Here $\psi$ is the angle from $\mathrm{X}$-rays impinging on point $A$ to $\mathrm{X}$-rays impinging on point $B$, given by

$$
\psi=\frac{T}{p} \frac{\sin 2 \theta_{\mathrm{B}}}{\cos \left(\chi-\theta_{\mathrm{B}}\right)},
$$

where $p$ is the distance from the $\mathrm{X}$-ray source to the crystal [see Appendix $B 1$ for the derivation of equation (8)].

The parameter $\Delta \phi$ should be adjusted from $\Delta \phi_{\infty}$ with

$$
\begin{aligned}
\Delta \phi & =\Delta \phi_{\infty}-\psi \\
& =-\frac{T}{R} \tan \left(\theta_{\mathrm{B}}-\chi\right)-\frac{T}{p} \frac{\sin 2 \theta_{\mathrm{B}}}{\cos \left(\chi-\theta_{\mathrm{B}}\right)} .
\end{aligned}
$$

(See Appendix B.)

The magic condition concept equation considering the source distance is

$$
\Delta \phi-\Delta \chi=\Delta \theta_{\mathrm{B}_{01}}
$$

or

$$
\frac{1+v}{2} \frac{T}{R} \sin 2 \chi-\frac{T}{R} \tan \left(\theta_{\mathrm{B}}-\chi\right)-\frac{1}{2} \frac{T}{p} \frac{\sin 2 \theta_{\mathrm{B}}}{\cos \left(\chi-\theta_{\mathrm{B}}\right)}=0 .
$$

Note that $R$ and $p$ can be either positive or negative. A positive $R$ corresponds to the convex side of the crystal facing the source, and a negative $R$ corresponds to the concave side of the crystal facing the source. A positive $p$ corresponds to a finite source distance or a diverging incident beam, and a negative $p$ corresponds to a 'beyond-infinite' source distance or a converging incident beam. When the source distance is infinite, equation (12) is equivalent to equation (6). Although equation (12) is independent of the thickness $T$, it has been kept in the equation, as it will help in understanding the situation when the equation is not equal to 0 [equation (13)]. The reduced version of this equation appears later as equation (19).

When the magic condition is not met, the residual, $\Delta \theta_{\mathrm{B}_{\text {mis }}}$, of equations (11) or (12) is the misalignment of the Bragg angles from matching the magic condition. The misalignment angle is given by

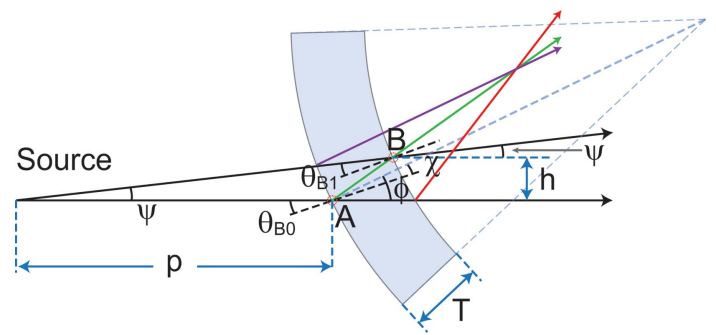

Figure 4

Magic condition with a finite source distance. 


$$
\begin{aligned}
\Delta \theta_{\mathrm{B}_{\text {mis }}} & =\Delta \phi-\Delta \chi-\Delta \theta_{\mathrm{B}_{01}} \\
& =\Delta \phi_{\infty}-\Delta \chi-\frac{\psi}{2} \\
& =\frac{T}{2}\left[\frac{1+v}{R} \sin 2 \chi-\frac{2}{R} \tan \left(\theta_{\mathrm{B}}-\chi\right)-\frac{1}{p} \frac{\sin 2 \theta_{\mathrm{B}}}{\cos \left(\chi-\theta_{\mathrm{B}}\right)}\right]
\end{aligned}
$$

\subsection{Equivalence with the other magic condition approach}

In previously published studies (Zhu et al., 2014; Martinson et al., 2015), a different method has been used to approach the magic condition. The matching of geometric focus, $q_{\mathrm{G}}$, and single-ray focus, $q_{\mathrm{S}}$, is used as the determinate condition (the focus-matching approach). The geometric focus is defined by (Schulze et al., 1998)

$$
\frac{\cos \left(\chi-\theta_{\mathrm{B}}\right)}{q_{\mathrm{G}}}-\frac{\cos \left(\chi+\theta_{\mathrm{B}}\right)}{p}=\frac{2}{R},
$$

and the single-ray focus is defined by (Martinson et al., 2015)

$$
q_{\mathrm{S}}=\frac{R \sin 2 \theta_{\mathrm{B}}}{2 \sin \left(\chi+\theta_{\mathrm{B}}\right)+(1+v) \sin 2 \chi \cos \left(\chi+\theta_{\mathrm{B}}\right)} .
$$

Note that Schulze et al. (1998) and Martinson et al. (2015) used sign conventions to handle different diffraction scenarios. With the definition of variables in the present work, equations (16) and (17) can be generalized to all scenarios, and thus the sign convention is not needed. By combining the two equations with $q_{\mathrm{S}}=q_{\mathrm{G}}$ and rearranging, the magic condition can be described as

$$
\begin{aligned}
& \frac{R}{2 p} \frac{\sin 2 \theta_{\mathrm{B}}}{\cos \left(\chi-\theta_{\mathrm{B}}\right)}-\frac{1+v}{2} \sin 2 \chi \\
& +\frac{\sin 2 \theta_{\mathrm{B}}}{\cos \left(\chi+\theta_{\mathrm{B}}\right) \cos \left(\chi-\theta_{\mathrm{B}}\right)}-\tan \left(\chi+\theta_{\mathrm{B}}\right)=0 .
\end{aligned}
$$

Rearranging the previous magic condition equation [equation (12)], we have

$$
\frac{R}{2 p} \frac{\sin 2 \theta_{\mathrm{B}}}{\cos \left(\chi-\theta_{\mathrm{B}}\right)}-\frac{1+v}{2} \sin 2 \chi+\tan \left(\theta_{\mathrm{B}}-\chi\right)=0 .
$$

Comparing equations (18) and (19) we see that the first two terms in the two equations are identical. It is not hard to get

$$
\frac{\sin 2 \theta_{\mathrm{B}}}{\cos \left(\chi+\theta_{\mathrm{B}}\right) \cos \left(\chi-\theta_{\mathrm{B}}\right)}-\tan \left(\chi+\theta_{\mathrm{B}}\right)=\tan \left(\theta_{\mathrm{B}}-\chi\right) .
$$

Thus, equations (18) and (19) are mathematically equivalent.

The angle-matching approach proposed in this work and the focus-matching approach presented previously describe the same optical phenomenon from different perspectives and are supportive to the correctness of each other. However, the focus-matching approach explains only the focal-size minimization and does not reveal the energy properties of the magic condition intuitively. The angle perspective to the magic condition explicitly describes the energy-related properties, forms a deeper understanding and enables more opportunities for the improvement of bent-Laue-crystal-related applications. This is discussed in more detail in Section 5.

\section{Energy resolution of a magic condition monochromator}

X-rays diffracted by a magic condition monochromator are sorted by angle and form a perfect fan beam. However, X-rays diffracted at the same Bragg angle are not purely monochromatic but contain X-rays with a range of energies, thus determining the energy resolution of the magic condition monochromator. This section will discuss the individual contributions to the energy resolution, the integration of the individual contributions and the ultimate energy resolution that is achievable under special conditions.

\subsection{Contributions to the energy resolution}

Firstly, the lattice spacing of the crystal is not uniform because of the crystal bending deformation. Diffracted X-rays exiting the crystal at the same point travel on the same path, but the $d$-spacing variation creates an energy spread in these $\mathrm{X}$-rays and the contribution can be described in the following equation:

$$
\left(\frac{\Delta E}{E}\right)_{1}=-\frac{\Delta d}{d}=\frac{T}{R}\left(\cos ^{2} \chi-v \sin ^{2} \chi\right) .
$$

Secondly, the X-ray source is at a finite distance. Thus, the incident angles of the X-rays vary when they hit the crystal at different locations in the diffraction plane. The contribution of the source-to-crystal distance to the energy spread can be described in the following equation:

$$
\left(\frac{\Delta E}{E}\right)_{2}=-\frac{\Delta \theta_{\mathrm{B}_{01}}}{\tan \theta_{\mathrm{B}}}=\frac{T}{p} \frac{\cos ^{2} \theta_{\mathrm{B}}}{\cos \left(\chi-\theta_{\mathrm{B}}\right)} .
$$

[See Appendix $C$ for the derivation of equations (21) and (22).]

Thirdly, the intrinsic Darwin width is the fundamental contribution to the energy spread in a diffracted X-ray beam. The contribution of the Darwin width can be described by

$$
\left(\frac{\Delta E}{E}\right)_{3}=\frac{w_{\mathrm{D}}}{\tan \theta_{\mathrm{B}}}
$$

where $w_{\mathrm{D}}$ is the Darwin width and is a constant value for a given reflection lattice (Zachariasen, 1945).

Fourthly, the source size is not infinitely small. Its contribution to the energy resolution is

$$
\left(\frac{\Delta E}{E}\right)_{4}=\frac{S}{p \tan \theta_{\mathrm{B}}}
$$

where $S$ is the size of the source transverse to the median ray in the diffraction plane.

In addition, the detector spatial resolution is a factor for the overall energy resolution. A smaller pixel size of the detector may give better energy resolution. Detector considerations are beyond the scope of the discussion for the monochromator here. 


\subsection{Integration of all contributions}

The first two contributions $\left[(\Delta E / E)_{1}\right.$ and $\left.(\Delta E / E)_{2}\right]$ to the energy resolution are directional. For the geometry in Fig. 4, because of Bragg's law, the lattice-spacing compression at point $B$ results in a higher energy than point $A$ for parallel incident X-rays. In the meantime, X-rays are incident at point $B$ with a shallower angle than at point $A$, which also corresponds to a higher energy for X-rays diffracted at point $B$. It is not hard to derive that the energy divergence between points $A$ and $B$ is the sum of the two terms and their values should be handled with signs. Therefore, the combined contribution of the first two terms to the energy resolution is

$$
\left(\frac{\Delta E}{E}\right)_{1 \& 2}=\left(\frac{\Delta E}{E}\right)_{1}+\left(\frac{\Delta E}{E}\right)_{2} .
$$

The interactions of these two contributions in different scenarios are explained in Appendix C3.

Assuming the source size is Gaussian distributed and is uncorrelated with the angular distribution, then the third and fourth contributions will add in quadrature with the other contributions. The overall energy resolution from all contributions should be put together in the following way:

$$
\begin{aligned}
\frac{\Delta E}{E}=\{ & {\left[\left(\frac{\Delta E}{E}\right)_{1}+\left(\frac{\Delta E}{E}\right)_{2}\right]^{2}+\left[\left(\frac{\Delta E}{E}\right)_{3}\right]^{2} } \\
& \left.+\left[\left(\frac{\Delta E}{E}\right)_{4}\right]^{2}\right\}^{1 / 2} .
\end{aligned}
$$

The simulated energy resolution for the magic condition geometry is shown in Fig. 5 (the focal distance is restrained for up to $10 \mathrm{~m}$ ). The lattice-spacing contribution is dominant when the focal distance is relatively close, as the crystal

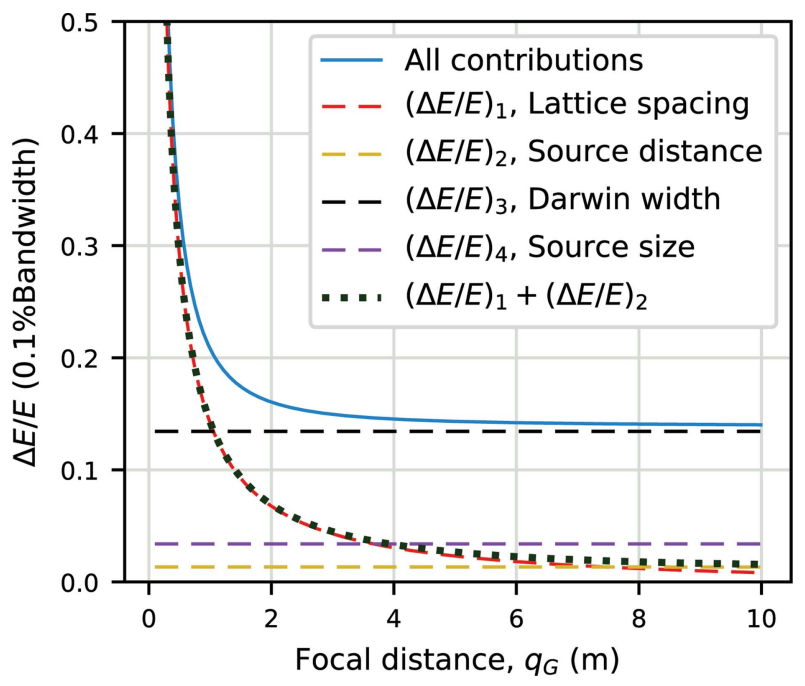

Figure 5

The total energy resolution and individual contributions for a magic condition monochromator in a range of focal distances, with $\mathrm{Si}(111)$ reflection, crystal thickness $T=0.3 \mathrm{~mm}$, source distance $p=22 \mathrm{~m}$, Poisson ratio $v=0.2$ and central X-ray energy $E=12.658 \mathrm{keV}$. The optimized $\chi$ angle for each $q_{\mathrm{G}}$ is calculated using equation (19). bending radius is small and causes more deformation of the crystal. The Darwin width contribution is constant for a given reflection and is the dominant term when the focal distance is relatively large, as the crystal deformation is not significant. The contributions of the source size and distance are usually small for a synchrotron X-ray source. The combined contributions of the two directional terms (lattice spacing and source distance) are dominated by the lattice spacing in the shown range of focal distance. A larger focal distance corresponds to a smaller bandwidth of the monochromator as the crystal bending radius is larger.

\subsection{The ultimate energy resolution magic condition}

Depending on the signs of $R$ and $p$, it is obvious that $(\Delta E / E)_{1}$ and $(\Delta E / E)_{2}$ can either add to or subtract from each other. When $R$ and $p$ are both positive (or both negative), $(\Delta E / E)_{1}$ and $(\Delta E / E)_{2}$ add to each other, and when one is positive and the other negative they are subtracted from one another. When $(\Delta E / E)_{1 \& 2}=0$, the energy blurring from $(\Delta E / E)_{1}$ and $(\Delta E / E)_{2}$ can be eliminated. With equations (21), (22) and (25), this condition is met when

$$
\frac{R}{p}=\frac{\left(\nu \sin ^{2} \chi-\cos ^{2} \chi\right) \cos \left(\chi-\theta_{\mathrm{B}}\right)}{\cos ^{2} \theta_{\mathrm{B}}} .
$$

When the magic condition equation [equation (12)] and the ultimate resolution equation [equation (27)] are both true, the energy resolution of a magic condition monochromator is only limited by the intrinsic Darwin width and the source size. For example, a pair of $\chi$ and $R$ can be obtained by solving equation (12) and equation (27) simultaneously for a given energy of interest and the remaining parameters.

For high-energy applications, equation (27) requires the bending radius of the crystal to be about equal to the source distance. The negative sign suggests that the source and the centre of the crystal bending circle are on the same side of the crystal, which implies that the ultimate energy resolution magic condition can not be met for a focusing crystal. For example, with a $22.0 \mathrm{~m}$ source distance and Si(111)-type reflection, the ultimate resolution magic condition for $12.658 \mathrm{keV} \mathrm{X}$-rays is met when $\chi$ is $0^{\circ}$ and $R$ is $-22.3 \mathrm{~m}$. The negative $R$ indicates that the source is on the concave side of the crystal and the diffracted beam is diverging. The Cauchois (1932) condition is satisfied and a specific choice of $\chi$ ensures the ultimate energy resolution. This condition is useful for a meridionally bent Laue crystal monochromator with higher energy resolution. Another matching case is used in a beam expander (Martinson et al., 2015) or other multiple-crystal systems with short (virtual) focal lengths. For example, with a $1.0 \mathrm{~m}$ source distance and other parameters being the same, the condition is met when $\chi$ is $0^{\circ}$ and $R$ is $-1.0 \mathrm{~m}$.

\section{Quasi-monochromatic beam for off-magic condition}

\subsection{Concept}

When the crystal does not match the magic condition, which is the general case for a bent Laue crystal, diffracted X-rays 
exiting at point $B$ no longer propagate on the same path as from point $A$ but form a fan beam with a deviation angle, as shown in Fig. 6. This deviation angle, $\Theta$, is the result of the misalignment of diffraction angles $\theta_{\mathrm{B}_{0}}$ and $\theta_{\mathrm{B}_{1}}$ at points $A$ and $B$, respectively:

$$
\begin{aligned}
\Theta & =2 \theta_{\mathrm{B}_{\mathrm{mis}}} \\
& =T\left[\frac{1+v}{R} \sin 2 \chi-\frac{2}{R} \tan \left(\theta_{\mathrm{B}}-\chi\right)-\frac{1}{p} \frac{\sin 2 \theta_{\mathrm{B}}}{\cos \left(\chi-\theta_{\mathrm{B}}\right)}\right] .
\end{aligned}
$$

$\Theta$ is directional and counterclockwise is defined as positive. Fig. 6 shows the scenario where $\theta_{\mathrm{B}_{1}}$ is smaller than the magic condition diffraction angle, and hence the deviation angle, $\Theta$, is below the 'reference ray', $A A^{\prime}$ ( $\Theta$ is negative). From point $B$ downwards along the downstream surface of the crystal, the diffraction angle gets larger and larger, and eventually a point $C$ can be found where $\theta_{\mathrm{B}_{2}}$, the diffraction angle at point $C$, equals the magic condition diffraction angle and the diffracted rays are parallel to the reference ray. Again, when the source is infinitely far away, $\theta_{\mathrm{B}_{2}}=\theta_{\mathrm{B}_{0}}$; otherwise, $\theta_{\mathrm{B}_{0}}$ and $\theta_{\mathrm{B}_{2}}$ differ by a correction for the finite source distance. Therefore, no matter whether the magic condition is met or not, there are always a series of diffracted X-rays parallel to the diffracted reference X-ray. The magic condition is a special case when all these parallel rays travel on the same path and exit the crystal at the same location. When the magic condition is not met, these parallel rays do not travel on the same path but exit the crystal at a continuous series of locations on the crystal surface and form a parallel beam (between $A A^{\prime}$ and $C C^{\prime}$ in Fig. 6) with a width not equal to zero. The term 'quasi-monochromatic beam' or 'quasi-mono beam' is used to represent this beam parallel to the diffracted reference ray. The phrase 'quasi' is used because 'monochromatic' is only true for this beam when the lattice-spacing variation, source distance, source size and intrinsic crystal Darwin width are neglected.

We should note that every ray diffracted at the upstream surface of the crystal (we name these rays the 'surfacediffracted X-rays') can be viewed as a reference ray, similar to $A A^{\prime}$ in Fig. 6. Each of these surface-diffracted X-rays will be accompanied by a quasi-mono beam when it leaves the crystal. Therefore, the entire diffracted beam can be treated methodologically as the sum of all the surface-diffracted X-rays and their companion quasi-mono beams. The magic condition is a

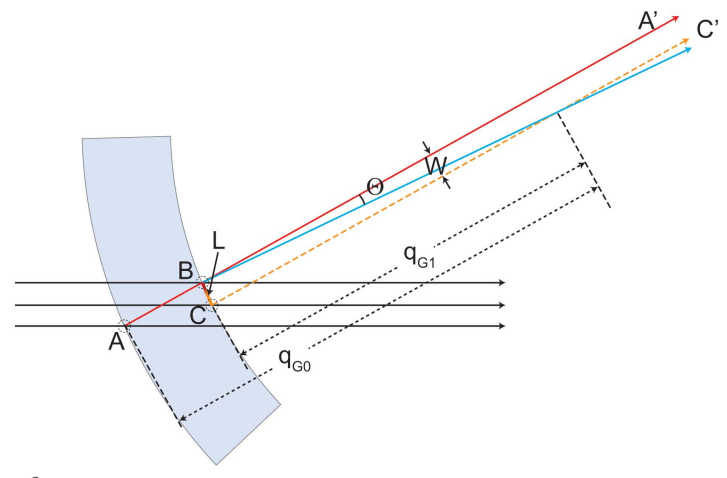

Figure 6

Geometry for the quasi-mono-beam width calculation. special case when the width of the quasi-mono beam is zero. The quasi-mono beam is a useful concept for interpreting bent Laue crystal optics in terms of focusing geometry and energy properties.

In the following, both infinite and finite source distance versions of the relevant parameters are given. The infinite version helps to understand the bulk of the behaviour as, in most instances, the source-to-crystal distance is large.

\subsection{Quasi-mono-beam width}

For a source infinitely far away, the arc length $L$ between points $B$ and $C$ corresponds to the diffraction angle divergence that amounts to half of the deviation angle, $\Theta$, as shown in Fig. 6. For a finite source distance, the $\mathrm{X}$-ray incident angles at points $B$ and $C$ are different. Alternatively, an easy way to find point $C$ is to draw a straight line parallel to the reference ray and through the geometric focus (caused by the downstream surface of the crystal to be accurate). Hence, the two edges of the quasi-mono beam are defined by the diffracted X-rays at points $A$ and $C$, or $A A^{\prime}$ and $C C^{\prime}$.

The width of the quasi-mono beam is given by

$$
\begin{aligned}
W= & q_{\mathrm{G}_{1}} \Theta \\
\simeq & q_{\mathrm{G}_{0}} \Theta \\
= & \frac{T}{(2 p / R)+\cos \left(\chi_{0}+\theta_{\mathrm{B}_{0}}\right)} \\
& \times\left[(1+v) \frac{p}{R} \sin 2 \chi_{0} \cos \left(\theta_{\mathrm{B}_{0}}-\chi_{0}\right)\right. \\
& \left.-\frac{2 p}{R} \sin \left(\theta_{\mathrm{B}_{0}}-\chi_{0}\right)-\sin 2 \theta_{\mathrm{B}_{0}}\right],
\end{aligned}
$$

where $q_{\mathrm{G}_{0}}$ and $q_{\mathrm{G}_{1}}$ are geometric focal distances corresponding to the upstream and downstream crystal surfaces, respectively, and $\chi_{0}$ is the effective asymmetry angle at point $A$, which is equivalent to $\chi$ hereinafter.

With the source distance at infinity, $p \gg R$, the width of the quasi-mono beam, $W_{\infty}$, is given by

$$
W_{\infty} \simeq T\left[\frac{1+v}{2} \sin 2 \chi_{0} \cos \left(\theta_{\mathrm{B}_{0}}-\chi_{0}\right)-\sin \left(\theta_{\mathrm{B}_{0}}-\chi_{0}\right)\right] .
$$

For a symmetrically bent Laue crystal, $\chi_{0}=0$, with an infinite source distance, the width of the quasi-mono beam, $W_{\infty}^{\text {sym }}$, is given by

$$
W_{\infty}^{\text {sym }}=-T \sin \theta_{\mathrm{B}_{0}} .
$$

The arc length of the quasi-mono-beam footprint on the downstream side of the crystal is given by

$$
\begin{aligned}
L & =\frac{W}{\cos \left(\theta_{\mathrm{B}_{2}}-\chi_{2}\right)} \\
& \simeq \frac{W}{\cos \left(\theta_{\mathrm{B}_{0}}-\chi_{0}\right)},
\end{aligned}
$$

where the subscripts 0 and 2 refer to variables corresponding to points $A$ and $C$ in Fig. 6, respectively. For a more accurate calculation of $L$ with equation (34), please see the calculation of $\theta_{\mathrm{B}_{2}}$ in Appendix $B 7$. 
Using equations (32)-(35), we have

$$
L_{\infty} \simeq T\left[\frac{1+v}{2} \sin 2 \chi_{0}-\tan \left(\theta_{\mathrm{B}_{0}}-\chi_{0}\right)\right]
$$

and

$$
L_{\infty}^{\mathrm{sym}}=-T \tan \theta_{\mathrm{B}_{0}} .
$$

(See Appendix $F$ for a summary of important variables.)

\subsection{Focal size}

Because the quasi-mono-beam width is constant all the way from the monochromator to the detector, it is not hard to see that the focal size of a bent asymmetric Laue crystal for X-rays from a point source is the same as the size of the quasi-monobeam width. When the finite source size, $S$, is considered, the total focal size is given by

$$
S_{\mathrm{f}}=\left[\left(\frac{q_{\mathrm{G}} S}{p}\right)^{2}+\frac{W^{2}}{12}\right]^{1 / 2} .
$$

The source is treated as a Gaussian distribution and the quasimono beam is treated as a uniform distribution where the variance has been used to represent its effective Gaussian width.

Another approach to calculate the focal size is using the single-ray focal distance and the geometric focal distance - a two-focus approach (see Appendix $D$ ). The focal sizes calculated using equation (38) and the two-focus approach for a point source and a range of asymmetry angles are compared in Fig. 7. The results show excellent agreement between the two approaches, which supports the quasi-mono-beam description.

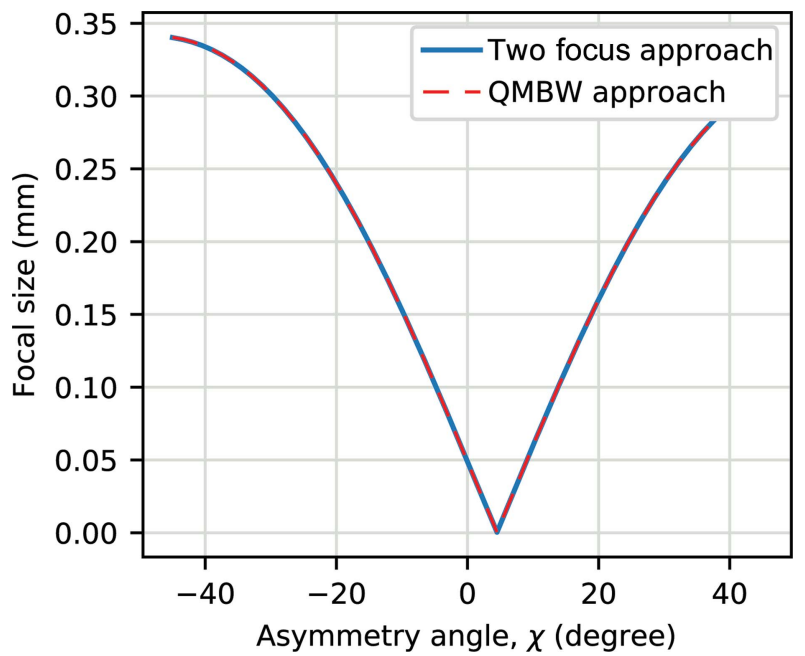

Figure 7

The calculated focal size against a range of asymmetry angles with two approaches: the focal distance geometry and the quasi-mono beam. The calculation was done for $\mathrm{Si}(111)$ with thickness $T=0.3 \mathrm{~mm}$, photon energy $E=12.658 \mathrm{keV}$, source distance $p=22 \mathrm{~m}$ and crystal bending radius $R=2 \mathrm{~m}$.

\subsection{Energy spread in the quasi-mono beam}

The energy spread in the quasi-mono beam is similar to the energy resolution of the magic condition reference ray. The intrinsic Darwin width of the crystal and the size of the source blur the energy resolution of every ray. The energy spreads caused by the lattice-spacing variation and the source distance are directional. The combination of the last two makes the energy of the quasi-mono beam lower on one side and higher on the other.

The fractional energy spread caused by the lattice-spacing variation is

$$
\left(\frac{\Delta E}{E}\right)_{Q 1}=-\frac{\Delta d}{d}=\frac{T}{R}\left(\cos ^{2} \chi_{0}-v \sin ^{2} \chi_{0}\right) .
$$

The fractional energy spread caused by a finite source distance is

$$
\begin{aligned}
\left(\frac{\Delta E}{E}\right)_{Q 2} & =\frac{1}{2 \tan \theta_{\mathrm{B}_{0}}}\left[\frac{T \sin 2 \theta_{\mathrm{B}_{0}}}{p \cos \left(\chi_{0}-\theta_{\mathrm{B}_{0}}\right)}+\frac{L \cos \left(\chi_{2}+\theta_{\mathrm{B}_{2}}\right)}{p}\right] \\
& \simeq \frac{1}{2 \tan \theta_{\mathrm{B}_{0}}}\left[\frac{T \sin 2 \theta_{\mathrm{B}_{0}}}{p \cos \left(\chi_{0}-\theta_{\mathrm{B}_{0}}\right)}+\frac{L \cos \left(\chi_{0}+\theta_{\mathrm{B}_{0}}\right)}{p}\right] .
\end{aligned}
$$

(See Appendix E.)

Therefore, the directional part of the fractional energy spread is simply given by

$$
\left(\frac{\Delta E}{E}\right)_{Q}=\left(\frac{\Delta E}{E}\right)_{Q 1}+\left(\frac{\Delta E}{E}\right)_{Q 2},
$$

or

$$
\Delta E_{Q}=\Delta E_{Q 1}+\Delta E_{Q 2},
$$

showing the energy difference of one edge ( $C C^{\prime}$ in Fig. 6) relative to the reference edge $\left(A A^{\prime}\right.$ in Fig. 6) of the quasimono beam.

\subsection{Energy spread of the X-rays at a point on a detector}

We now consider a detector located at a distance $d_{\mathrm{GD}}$ from the geometric focus of the beam. In a general case, a single point on the detector sees overlapping quasi-mono beams, associated with a range of surface-diffracted X-rays. As an example, the point $C^{\prime}$ shown in Fig. 8(a) is illuminated by a series of quasi-mono beams (not shown in the figure), where QMB 1 and QMB 2 are the quasi-mono beams associated with the boundary surface-diffracted X-rays $A A^{\prime}$ and $D C^{\prime}$ diffracted from the upstream surface points $A$ and $D$, respectively. The point $C^{\prime}$ sees all the quasi-mono beams associated with surface-diffracted X-rays from all points between $A$ and $D$ along the upstream surface of the crystal. As a consequence, the energy spread of the X-rays at point $C^{\prime}$ is determined by the difference between the energies of the bottom edge $\left(C C^{\prime}\right)$ of QMB 1 and the top edge $\left(D C^{\prime}\right)$ of QMB 2. The energies of the X-rays in the other quasi-mono beams that hit point $C^{\prime}$ will be between the energies of ray $C C^{\prime}$ and ray $D C^{\prime}$.

Surface-diffracted X-rays between $D C^{\prime}$ and $A A^{\prime}$ form a fanshaped surface-diffracted beam, as shown in Fig. 8(b). As this 
surface-diffracted beam and the quasi-mono beam share $A A^{\prime}$ as the reference edge, the energy difference at point $C^{\prime}\left(\Delta E_{\mathrm{P}}\right)$ between $C C^{\prime}$ and $D C^{\prime}$ can be described by the difference between the energy change from point $A$ to point $D\left(\Delta E_{\mathrm{G}}\right)$ and that from point $A$ to point $C\left(\Delta E_{\mathrm{Q}}\right)$ :

$$
\Delta E_{\mathrm{P}}=\Delta E_{\mathrm{Q}}-\Delta E_{\mathrm{G}}
$$

Here $\Delta E_{\mathrm{Q}}$ is the energy spread of the quasi-mono beam described in Section 5.4 and $\Delta E_{\mathrm{G}}$ is the energy spread of the surface-diffracted fan beam bounded by $A A^{\prime}$ and $D C^{\prime} . \Delta E_{\mathrm{G}}$ in fractional form can be described with two parts: (i) $(\Delta E / E)_{\mathrm{G} 1}$, caused by the rotation of the Bragg planes $\left(\Delta \theta_{\mathrm{G} 1}\right)$ between $A$ and $D$ due to the bending of the crystal,

$$
\begin{aligned}
\left(\frac{\Delta E}{E}\right)_{\mathrm{G} 1} & =-\frac{\Delta \theta_{\mathrm{G} 1}}{\tan \theta_{\mathrm{B}_{0}}} \\
& =-\frac{L_{A D}}{R \tan \theta_{\mathrm{B}_{0}}},
\end{aligned}
$$

and (ii) $(\Delta E / E)_{\mathrm{G} 2}$, caused by the incident angle deviation ( $\Delta \theta_{\mathrm{G} 2}$ ) from the source to the points $A$ and $D$,

$$
\begin{aligned}
\left(\frac{\Delta E}{E}\right)_{\mathrm{G} 2} & =-\frac{\Delta \theta_{\mathrm{G} 2}}{\tan \theta_{\mathrm{B}_{0}}} \\
& =-\frac{L_{A D} \cos \left(\chi_{0}+\theta_{\mathrm{B}_{0}}\right)}{p \tan \theta_{\mathrm{B}_{0}}} .
\end{aligned}
$$

$L_{A D}$ is the arc length between points $A$ and $D$ or the footprint of the surface-diffracted beam on the crystal, which is given by

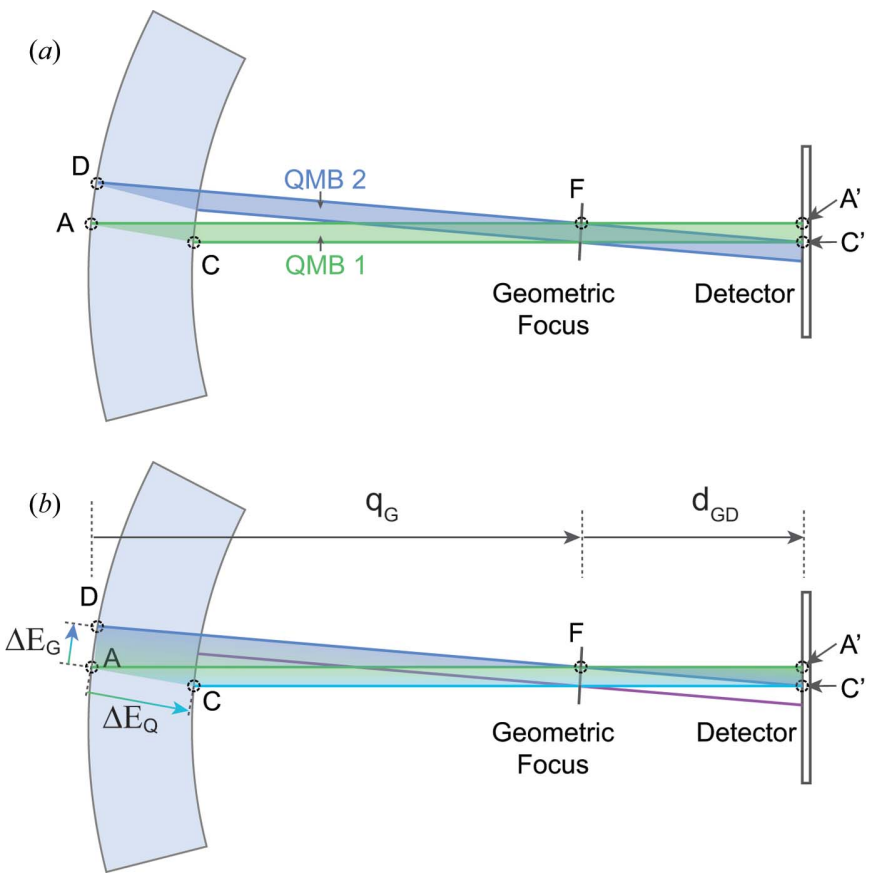

Figure 8

The energy spread of a point on a detector at a certain distance. (a) A point on the detector illuminated by two boundary quasi-mono beams (QMB 1 and QMB 2). (b) A quasi-mono beam and a surface-diffracted beam that illuminate the same area on the detector.

$$
\begin{aligned}
L_{A D} & =\frac{q_{\mathrm{G}} W}{d_{\mathrm{GD}} \cos \left(\chi_{0}-\theta_{\mathrm{B}_{0}}\right)} \\
& \simeq \frac{q_{\mathrm{G}} L}{d_{\mathrm{GD}}} .
\end{aligned}
$$

$d_{\mathrm{GD}}$ is the distance from the geometric focus to the detector.

The energy spread in the surface-diffracted beam is given by

$$
\begin{aligned}
\left(\frac{\Delta E}{E}\right)_{\mathrm{G}}= & \left(\frac{\Delta E}{E}\right)_{\mathrm{G} 1}+\left(\frac{\Delta E}{E}\right)_{\mathrm{G} 2} \\
= & -\frac{q_{\mathrm{G}}}{d_{\mathrm{GD}} \cos \left(\chi_{0}-\theta_{\mathrm{B}_{0}}\right) \tan \theta_{\mathrm{B}_{0}}} \\
& \times\left[\frac{W}{R}+\frac{W \cos \left(\chi_{0}+\theta_{\mathrm{B}_{0}}\right)}{p}\right] \\
\simeq & -\frac{q_{\mathrm{G}}}{d_{\mathrm{GD}} \tan \theta_{\mathrm{B}_{0}}}\left[\frac{L}{R}+\frac{L \cos \left(\chi_{0}+\theta_{\mathrm{B}_{0}}\right)}{p}\right],
\end{aligned}
$$

or

$$
\Delta E_{\mathrm{G}}=\Delta E_{\mathrm{G} 1}+\Delta E_{\mathrm{G} 2} .
$$

\subsection{Monochromatic focus}

The description of the energy spread of the X-rays at a point on a detector leads to an interesting special case when

$$
\Delta E_{\mathrm{P}}=\Delta E_{\mathrm{Q}}-\Delta E_{\mathrm{G}}=0 .
$$

This equation predicts the existence of the 'monochromatic focus', where X-rays of one energy intersect at a single point on the detector (the parameters taken into account are the lattice-spacing deformation and the source distance). When the detector is placed at the monochromatic focus, energyresolution widening caused by lattice-spacing deformation and the finite source distance can be neutralized by the crystal surface curvature oriented energy dispersion. The only remaining contributors to the energy-resolution widening will be the intrinsic crystal Darwin width and the source size. This is valuable because not only is the energy resolution improved significantly but also the remaining two contributors are constants related to the monochromator itself. This means the bent crystal will have a large bandwidth without compromising the energy resolution on the detector.

5.6.1. Derivation of the monochromatic focal distance. With a given X-ray source and monochromator, the energy spread of the quasi-mono beam $\left(\Delta E_{\mathrm{Q}}\right)$ is fixed and independent of the detector distance ( $\left.d_{\mathrm{GD}}\right)$ [equations (39), (40), (42) and (43)], while the energy spread of the surface-diffracted beam $\left(\Delta E_{\mathrm{G}}\right)$ is $d_{\mathrm{GD}}$ dependent [equation (53)]. Thus, the monochromatic focal distance can be approached by solving for $d_{\mathrm{GD}}$ in equation (55). The $d_{\mathrm{GD}}$ that satisfies equation (55), or the distance from the geometric focus to the monochromatic focus, will be referred to as the optimal detector distance, denoted $d_{\mathrm{GM}}$.

The existence of $d_{\mathrm{GM}}$ to fulfil equation (55) can be simply explained as follows. The quasi-mono beam is formed by parallel X-rays; its energy spread and energy spread per width are both unchanged on its way to the detector. Conversely, the fan-shaped surface-diffracted beam has its energy spread per 
width changing as the beam propagates. When a detector is placed at a special distance from the geometric focus $\left(d_{\mathrm{GM}}\right.$ in Fig. 9), the energy spreads per width of the two beams on the detector can be aligned with each other perfectly, as they share a common reference ray. Hence, a 'monochromatic focus' ( $C^{\prime}$ in Fig. 9) is formed at this location, where the X-rays arriving at the same point on the detector will have the same energy.

Mathematically, $d_{\mathrm{GM}}$ is solved from equation (55) to be

$$
d_{\mathrm{GM}}=-\frac{q_{\mathrm{G}}}{(\Delta E / E)_{Q} \tan \theta_{\mathrm{B}_{0}}}\left[\frac{L}{R}+\frac{L \cos \left(\chi_{0}+\theta_{\mathrm{B}_{0}}\right)}{p}\right],
$$

or

$$
\begin{aligned}
d_{\mathrm{GM}}= & -\frac{q_{\mathrm{G}}}{(\Delta E / E)_{\mathrm{Q}} \tan \theta_{\mathrm{B}_{0}} \cos \left(\chi_{0}-\theta_{\mathrm{B}_{0}}\right)} \\
& \times\left[\frac{W}{R}+\frac{W \cos \left(\chi_{0}+\theta_{\mathrm{B}_{0}}\right)}{p}\right] .
\end{aligned}
$$

The monochromatic focal distance is simply given by

$$
q_{\mathrm{M}}=q_{\mathrm{G}}+d_{\mathrm{GM}} .
$$

When the source distance is infinite $(p \gg R)$, the optimal focus-to-detector distance, $d_{\mathrm{GM} \infty}$, is given by

$$
\begin{aligned}
d_{\mathrm{GM} \infty} & =-\frac{q_{\mathrm{G} \infty}}{(\Delta E / E)_{\mathrm{Q} \infty} \tan \theta_{\mathrm{B}_{0}}} \frac{L_{\infty}}{R} \\
& =\frac{R(1+v) \cos \left(\theta_{\mathrm{B}_{0}}-\chi_{0}\right) \sin 2 \chi_{0}-2 R \sin \left(\theta_{\mathrm{B}_{0}}-\chi_{0}\right)}{4\left(v \sin ^{2} \chi_{0}-\cos ^{2} \chi_{0}\right) \tan \theta_{\mathrm{B}_{0}}} .
\end{aligned}
$$

For a symmetric bent Laue crystal $(\chi=0)$ with infinite source distance, the geometric focus to monochromatic focus distance, $d_{\mathrm{GM} \infty}^{\mathrm{sym}}$, is given by

$$
d_{\mathrm{GM} \infty}^{\mathrm{sym}}=\frac{R \cos \theta_{\mathrm{B}}}{2} \equiv q_{\mathrm{G} \infty}^{\mathrm{sym}},
$$

where $q_{\mathrm{G} \infty}^{\mathrm{sym}}$ is the geometric focal distance when $\chi=0$ and $p \gg R$.

The monochromatic focal distance at this condition is then

$$
q_{\mathrm{M} \infty}^{\mathrm{sym}}=2 q_{\mathrm{G} \infty}^{\mathrm{sym}}=R \cos \theta_{\mathrm{B}} .
$$

The existence of the monochromatic focus can be described in the following way as well. For a fixed $\lambda$ in $\lambda=2 d_{h k l} \sin \theta$, it requires a recombination of the variations in lattice spacing, $\delta d_{h k l}$, and Bragg angle, $\delta \theta$, given by

$$
\begin{gathered}
\delta \lambda=2 \sin \theta \delta d_{h k l}+2 d_{h k l} \cos \theta \delta \theta \equiv 0, \\
\delta \theta=-\tan \theta \frac{\delta d_{h k l}}{d_{h k l}} .
\end{gathered}
$$

For a fixed curvature of the bent crystal, there is a continuous distribution in lattice spacings from the compressed side to the expanded side. Because of the curvature of the crystal, there will be planes where the variation in angle given by equation (64) is met. Therefore, there is a continuous set of diffracted beams, all with the same energy. And since there is a range of diffracted beam angles, this set of rays will focus somewhere, at either a real or a virtual focus.

5.6.2. The monochromatic focus and the magic condition. The magic condition is a special case of bent Laue crystal optics when the quasi-mono-beam width is zero and, therefore, the
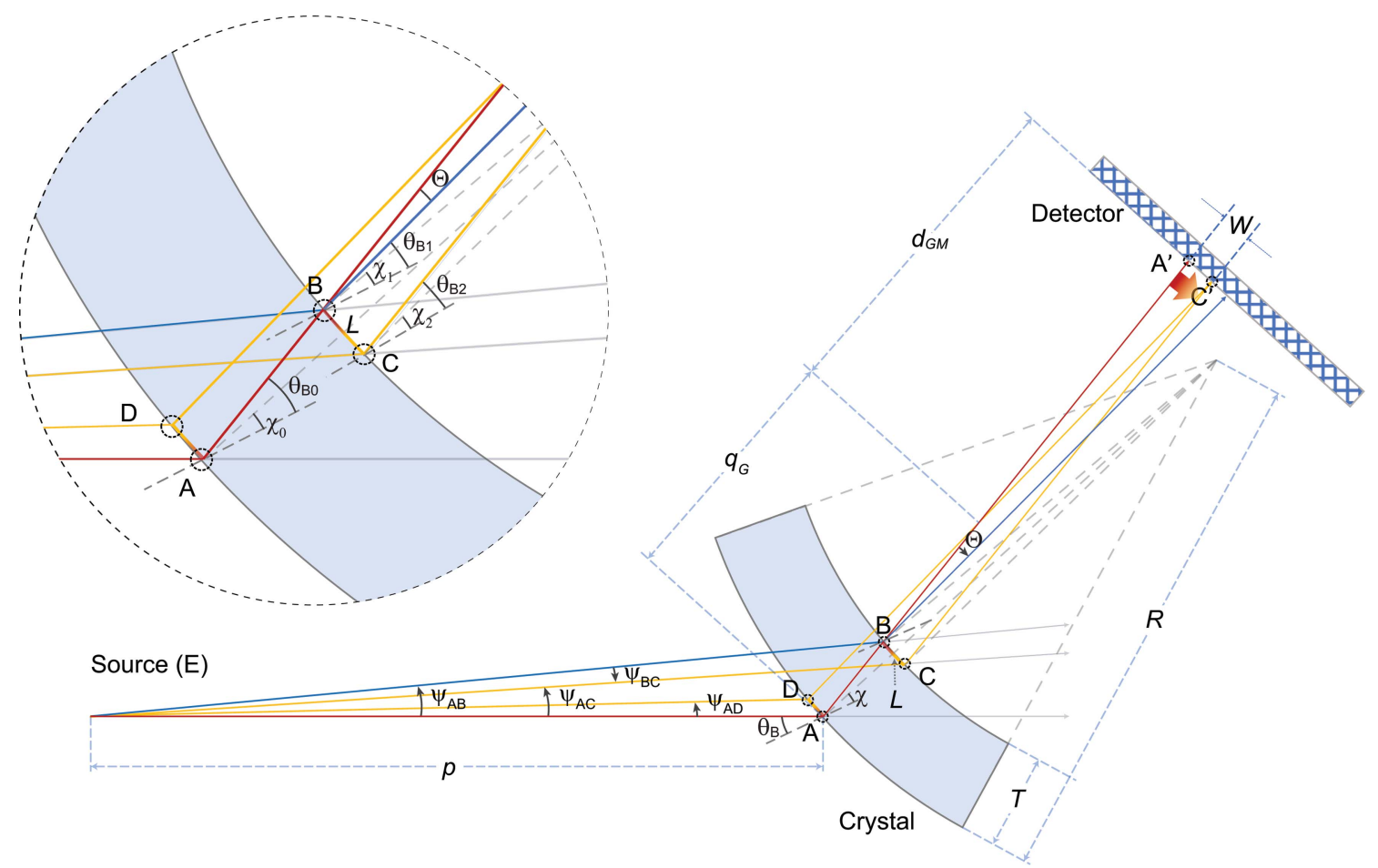

Figure 9

Quasi-monochromatic beam geometry. 
energy spread of the quasi-mono beam is compressed into a spatial width of zero. To find the monochromatic focus for the magic condition geometry, the surface-diffracted beam has to have the same energy spread with a zero width, which can only be satisfied at the geometric focus or $d_{\mathrm{GM}}=0$. This can also be seen from equation (57) by setting the quasi-mono-beam width $W=0$.

Another extreme case is when the energy spread of the quasi-mono beam is zero. The monochromatic focus can be found at infinite distance. This can also be seen from equation (57) by setting the energy spread of the quasi-mono beam $(\Delta E / E)_{\mathrm{Q}}=0$.

The problem that arises from the magic condition monochromatic focus is that all energies diffracted by the curved crystal also arrive at this location. To have a situation where the monochromatic focus results in spatially separated locations in a detector requires that the magic condition not be met. The only exception is that the magic condition is met while the 'quasi-mono' beam is monochromatic $\left[(\Delta E / E)_{\mathrm{Q}}=0\right]$. In this case, the monochromatic focus is everywhere in the diffracted beam (this is also the special case discussed in Section 4.3).

In summary, when the magic condition is met, the monochromatic focus is at the geometric focus $\left(d_{\mathrm{GM}}=0\right)$. When the quasi-mono beam is monochromatic, the monochromatic focus is at infinity $\left(d_{\mathrm{GM}}=\infty\right)$. When both conditions are met, the monochromatic focus is everywhere between the geometric focus and infinity.

When the crystal diffraction geometry misses the magic condition by a small amount, the energy-spread direction of the quasi-mono beam can be either in the same direction or opposite to the energy spread of the surface-diffracted beam. When the energy spreads are in the same (opposite) direction, the quasi-mono beam can improve (degrade) the energy resolution compared with a magic condition monochromator. The ultimate improvement arises when the energy spread of the quasi-mono beam perfectly matches that of the surfacediffracted beam.

5.6.3. Characteristics of the monochromatic focus. When the detector is placed at the monochromatic focus, the only contribution to the widening of the energy resolution is from the intrinsic Darwin width of the crystal and the source size. For a given monochromator, the monochromatic focal distance is energy dependent. An example is shown in Fig. 10(a) for an $\mathrm{Si}(022)$ monochromator with $-15.8^{\circ}$ asymmetry angle, $2 \mathrm{~m}$ bending radius and $22 \mathrm{~m}$ source distance. (Again, the contribution to the energy resolution from the effective detector pixel size is put aside from the discussion in this work.)

For a particular energy of interest and tunable asymmetry angle, the relation between the asymmetry angle and the optimal focus-to-detector distance is shown in Fig. 10(b). The optimal focus-to-detector distance is found to be positive between the magic condition $\chi$ angle (green dashed line) and the singularity $\chi$ angle (orange dashed line). The singularity $\chi$ angle corresponds to the directional energy spread being zero in the quasi-mono beam.
Given the beam diffracted by a bent crystal contains X-rays with a range of energies, a detector placed at the monochromatic focus of one energy would miss the monochromatic foci of all the other energies. The trajectory of the monochromatic foci of all energies for an example monochromator with an incident beam of $1 \mathrm{~cm}$ in height is shown in Fig. 11(a).

However, the monochromatic foci of all energies of the diffracted X-rays are close to this position, and the energy widths at all pixels illuminated by the diffracted beam are still close to zero (neglecting the crystal's intrinsic Darwin width and the source-size effect), as shown in Fig. 11(b). The energy widths when the (geometric) focus-to-detector distance equals the geometric focal distance is shown as a comparison. The correlation of energy widths and focus-to-detector distances for X-rays with certain energies is demonstrated in Fig. 11(c), where the energy widths are minimized at the monochromatic foci.

Therefore, by simply placing the detector at the monochromatic focus when applicable, existing bent Laue $K$-edge subtraction imaging systems may gain the capability for highenergy-resolution applications [e.g. spectral $K$-edge subtraction imaging (Zhu et al., 2014) and wide-field energydispersive X-ray absorption spectroscopy imaging (Qi, Shi et al., 2019)]. Note, however, that the detector distance for

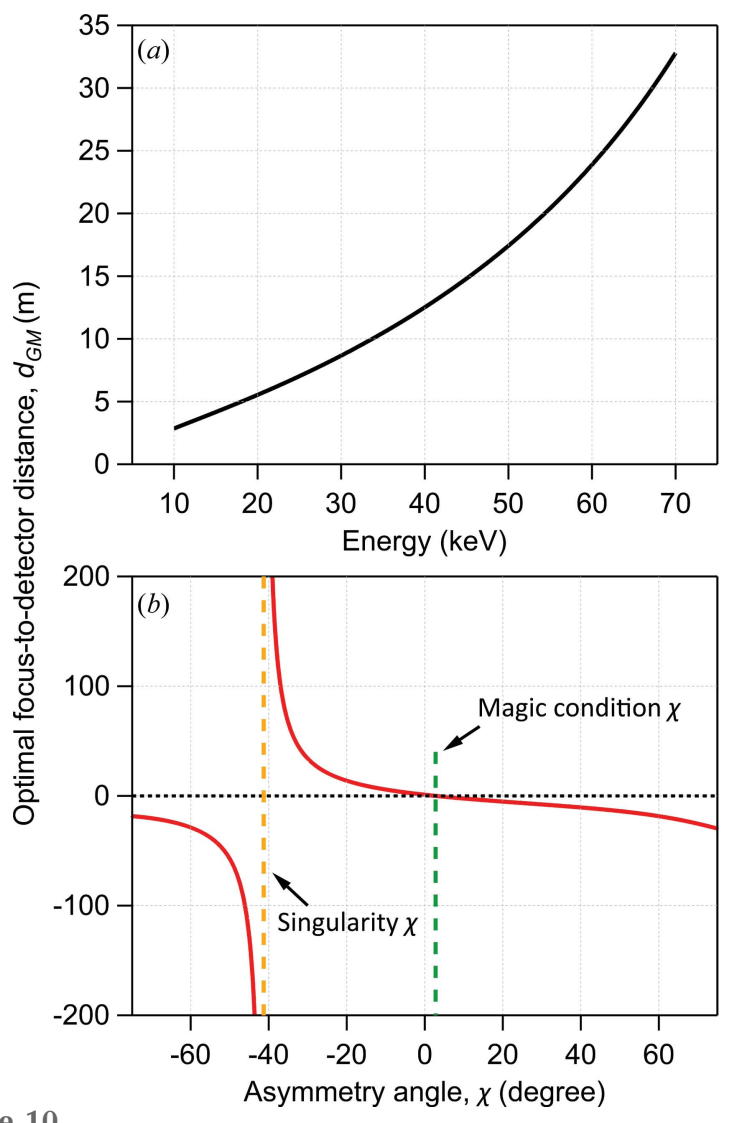

Figure 10

The optimal detector distance $d_{\mathrm{GM}}$ for an $\mathrm{Si}(022)$ reflection in a [511]oriented crystal with source distance $p=22 \mathrm{~m}$ and bending radius $R=$ $2 \mathrm{~m}$. (a) The optimal distance in a range of energies with the asymmetry angle $\chi=-15.8^{\circ}$. (b) The optimal distance $d_{\mathrm{GM}}$ as a function of asymmetry angle $\chi$ at a photon energy $E=33.169 \mathrm{keV}$. 

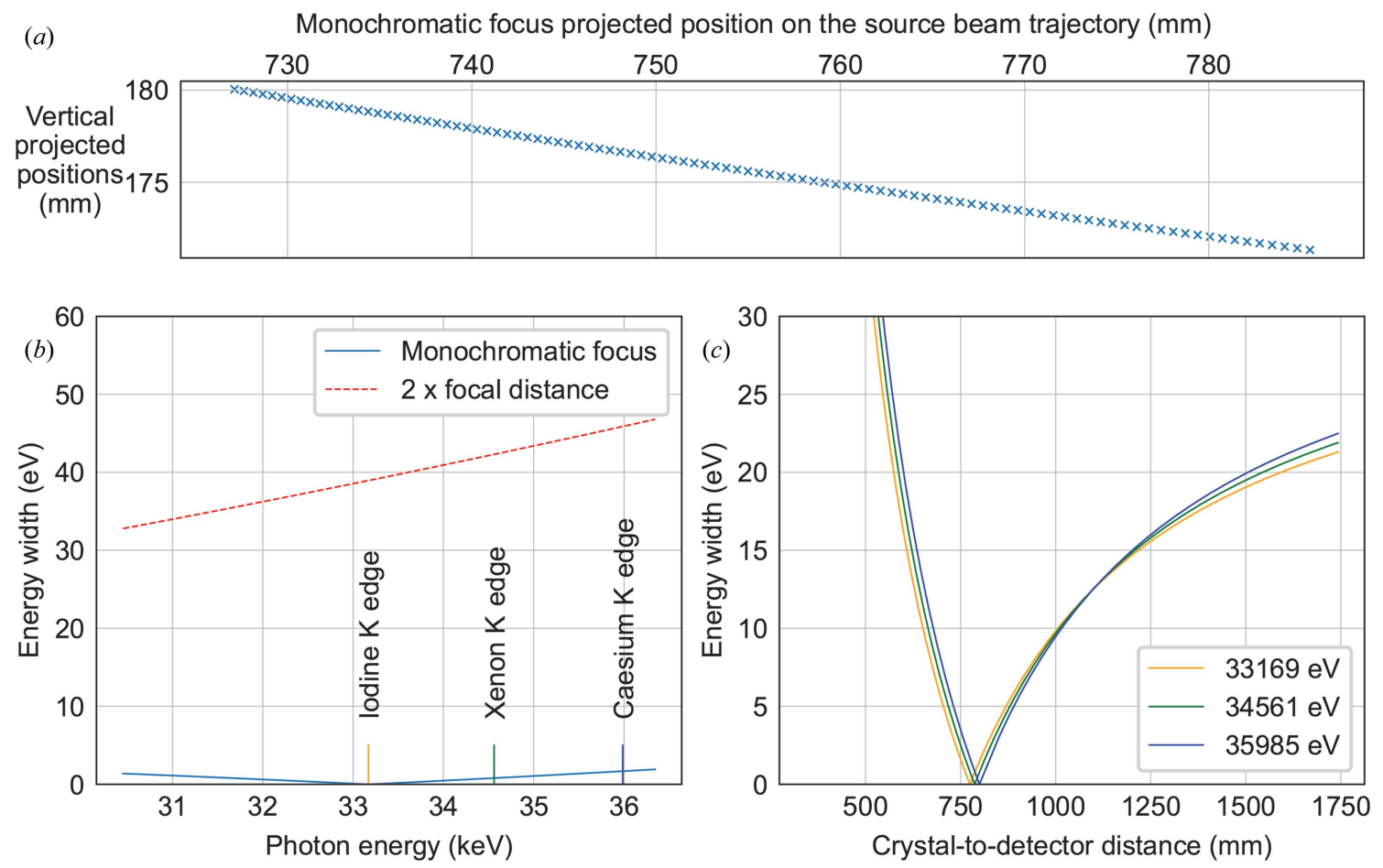

Figure 11

An example of the monochromatic focus behaviour for an $\mathrm{Si}(311)$ monochromator with bending radius $R=0.5 \mathrm{~m}$, thickness $T=0.5 \mathrm{~mm}$, asymmetry angle $\chi=3.33^{\circ}$, source distance $p=22 \mathrm{~m}$ and incident-beam height simulated as $10 \mathrm{~mm}$. ( $a$ ) The trajectory of monochromatic foci of all energies in the diffracted beam (low to high energy is left to right). The horizontal and vertical axes are monochromatic focus positions projected onto the incidentbeam axis and its normal axis in the diffraction plane, respectively. The vertical and horizontal positions are relative to the location where the central incident X-ray enters the crystal. $(b)$ Energy widths at corresponding energy-pixel positions when the detector is placed at the central ray monochromatic focus and at a distance twice the focal distance. (c) Central ray (at $K$-edge energies of iodine, xenon and caesium) energy widths for a range of focus-to-detector distances.

optimal energy resolution may be, and probably is, not accessible in many cases.

5.6.4. Clarification of another type of monochromatic focus. The concept of another type of 'monochromatic focus' has been described by Tolentino et al. $(1988,1989)$ where the focus is located on the Rowland circle of the crystal for corresponding monochromatic X-rays. At this spot, the image size of the source is zero for these monochromatic X-rays, and therefore the size of the source has zero contribution to the blurring of the energy. Tolentino's monochromatic focus does not account for the crystal lattice deformation caused by the bending of the crystal and works better for a Bragg-type monochromator than the Laue type, because in a bent Lauetype monochromator, the lattice spacing deformation needs to be taken into account, and it makes a much greater contribution (inversely proportional to the crystal bending radius) than the source-size-related contribution to the energy blurring. Another limitation to Tolentino's monochromatic focus is that, to fulfil the Rowland circle geometry, the $\mathrm{X}$-ray source has to be on the concave side of the crystal, which is not suitable for a focusing Laue monochromator where the convex side of the crystal is facing the source.

Whether the new monochromatic focus and Tolentino's monochromatic focus can be put together will be studied in future work.

\section{Discussion and conclusions}

This paper describes the general behaviour of bent Laue crystals from a ray-tracing point of view. Although this has been covered extensively in previous work, there is a need to decouple the description of the spatial focal properties of these crystals from the diffracted-beam energies of the rays.

The analysis in this paper has introduced a parallel quasimonochromatic beam in the X-rays diffracted by the bent crystal. It interprets the bent-crystal diffraction in terms of rays diffracted at the crystal surface and their companion quasi-mono beams. This quasi-mono beam leads to a deeper understanding of bent-crystal diffraction because it (i) builds an intuitive view of bent-crystal diffraction, (ii) explains both the energy and spatial properties of the diffracted beam, (iii) predicts phenomena that can improve energy-dispersionrelated X-ray imaging techniques, and (iv) provides a powerful tool for easier realization of ray-tracing simulations for bent Laue monochromators.

This new approach explains the magic condition, which gives the requirements for achieving a small spatial focus and good energy resolution, as a special case when the width of the quasi-mono beam is zero and, more importantly, explains the focusing behaviour of bent crystals when the magic condition is not met. This allows one to predict the more realistic diffraction conditions encountered experimentally. 


\section{APPENDIX $A$}

\section{$\Delta \chi$ and $\Delta \phi$}

Due to the bending force, the crystal is compressed on the concave side and expanded on the convex side in the direction of the $Y$ axis, as shown in Fig. 12. The compression or expansion causes an opposite deformation in the direction of the $X$ and $Z$ axes. The $Z$ axis (not shown in the figure) is orthogonal to the plane of $X Y$ and is not considered in the discussion here. The thickness of the crystal (along the $X$ axis) is changed by bending considering the elastic property of the crystal through the Poisson ratio, which is defined by

$$
v=-\frac{\Delta X / X}{\Delta Y / Y}
$$

The negative sign implies that the compression (expansion) $\Delta Y$ results in a transverse expansion (compression) $\Delta X$. Thus, a crystal when bent will experience a growth in thickness on the compressed concave side and a reduction in thickness on the expanded convex side. A crystal with original thickness $T_{0}$ has a thickness on the compressed side of the neutral plane of $\tau_{+}$, a thickness on the expanded side of $\tau_{-}$and a total bent thickness $T$ for the discussion hereafter.

\section{A1. $\Delta \chi$}

The change in $\chi$ over the crystal thickness is also a result of the deformation of the crystal as shown in Fig. 12. Assuming in the first unit cell (the left-hand blue-shaded box) $\tan \chi_{0}=h_{0} / t_{0}$, then in the last unit cell (the right-hand side),

$$
\begin{aligned}
& h=h_{0}\left(1-\tau_{+} / R\right), \\
& t=t_{0}\left(1+v \tau_{+} / R\right) .
\end{aligned}
$$

Therefore,

$$
\tan (\chi+\Delta \chi)=\frac{h}{t}=\frac{1-\tau_{+} / R}{1+\nu \tau_{+} / R} \frac{h_{0}}{t_{0}}=\frac{1-\tau_{+} / R}{1+\nu \tau_{+} / R} \tan \chi_{0} .
$$

Then

$$
\frac{\tan \chi_{0}+\tan \Delta \chi}{1-\tan \chi_{0} \tan \Delta \chi}=\frac{1-\tau_{+} / R}{1+\nu \tau_{+} / R} \tan \chi_{0} .
$$

Solving the equation using the small-angle approximation for $\Delta \chi$, we get

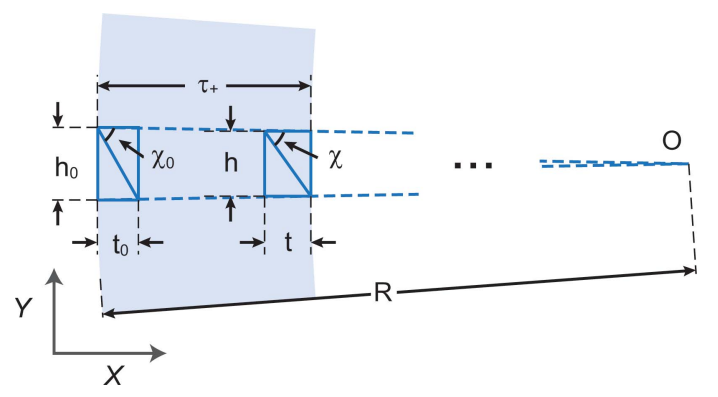

Figure 12

A schematic diagram to derive the change in $\chi$.

$$
\Delta \chi_{+}=\tan \Delta \chi=\frac{-(1+v) \tau_{+} \tan \chi_{0}}{\left(R+v \tau_{+}\right)+\left(R-\tau_{+}\right) \tan ^{2} \chi_{0}} .
$$

This can be generalized to the expansion side of the neutral plane as

$$
\Delta \chi_{-}=\tan \Delta \chi=\frac{(1+v) \tau_{-} \tan \chi_{0}}{\left(R-v \tau_{-}\right)+\left(R+\tau_{-}\right) \tan ^{2} \chi_{0}} .
$$

The total change in $\chi$ across the bent crystal is then

$$
\begin{aligned}
\Delta \chi & =\Delta \chi_{+}-\Delta \chi_{-} \\
& \simeq-\frac{\tau_{+}+\tau_{-}}{2 R}(1+v) \sin 2 \chi_{0} \\
& =-\frac{T(1+v)}{2 R} \sin 2 \chi_{0} .
\end{aligned}
$$

\section{A2. $\Delta \phi$}

$\phi$ is defined as the directional angle from the transmitted $\mathrm{X}$-ray to the crystal surface normal (positive for counterclockwise), and the subscripts 0 and 1 correspond to the points $A$ and $B$, respectively, in Fig. 13, which shows again the compressed half of the crystal. $O$ is the centre of the crystal bending circle. $A O$ and $B O$ are along the corresponding crystal surface normal directions, and $A B$ is the path of the diffracted X-ray in the crystal.

$\Delta \phi$, the change in $\phi$, is defined as $\phi_{1}-\phi_{0}$. It is obvious that $\Delta \phi$ is equal to the negative of the angle $\omega$ as shown in Fig. 13:

$$
\omega=\phi_{0}-\phi_{1}=-\Delta \phi \text {. }
$$

In the triangle $A B O$,

$$
a+b=R,
$$

where

$$
\begin{gathered}
a=\frac{c}{\tan \angle B A O}=\frac{c}{\tan \left(\theta_{\mathrm{B}}-\chi\right)}=\frac{\left(R-\tau_{+}\right) \sin \omega}{\tan \left(\theta_{\mathrm{B}}-\chi\right)}, \\
b=\left(R-\tau_{+}\right) \cos \omega .
\end{gathered}
$$

Therefore,

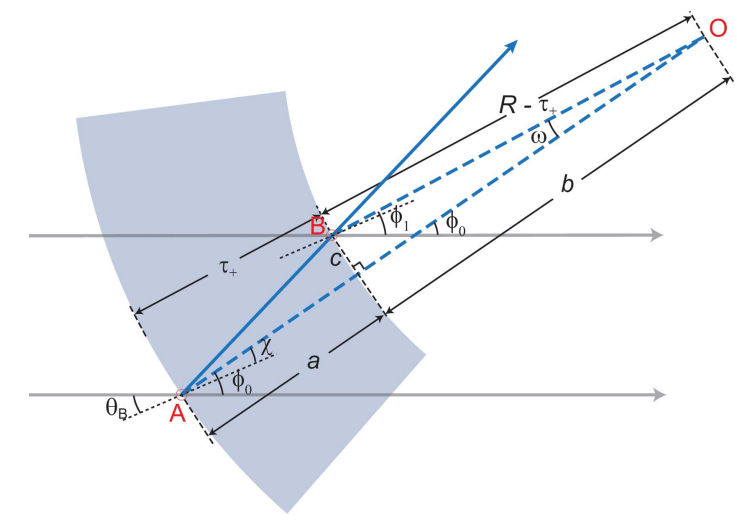

Figure 13

A schematic diagram to derive the change in $\phi$. Points $A$ and $B$ are the same as in Fig. 2. The relevant dimensions and angles are discussed in the text. 


$$
\frac{\left(R-\tau_{+}\right) \sin \omega}{\tan \left(\theta_{\mathrm{B}}-\chi\right)}+\left(R-\tau_{+}\right) \cos \omega=R .
$$

Using the small-angle approximation, we have

$$
\frac{\left(R-\tau_{+}\right) \omega}{\tan \left(\theta_{\mathrm{B}}-\chi\right)}+\left(R-\tau_{+}\right)\left(1-\frac{\omega^{2}}{2}\right)=R .
$$

Solving the equation above for $\omega$, we get two solutions:

$$
\omega_{1}=\frac{1}{\tan \left(\theta_{\mathrm{B}}-\chi\right)}-\left\{\frac{1}{\left[\tan \left(\theta_{\mathrm{B}}-\chi\right)\right]^{2}}-\frac{2 \tau_{+}}{R-\tau_{+}}\right\}^{1 / 2}
$$

and

$$
\omega_{2}=\frac{1}{\tan \left(\theta_{\mathrm{B}}-\chi\right)}+\left\{\frac{1}{\left[\tan \left(\theta_{\mathrm{B}}-\chi\right)\right]^{2}}-\frac{2 \tau_{+}}{R-\tau_{+}}\right\}^{1 / 2} .
$$

The first solution, $\omega_{1}$, is the one we need, while $\omega_{2}$ exists mathematically but is not of practical use for the optical application here. Thus, for the compression side of the crystal,

$$
\Delta \phi_{+}=\left\{\frac{1}{\left[\tan \left(\theta_{\mathrm{B}}-\chi\right)\right]^{2}}-\frac{2 \tau_{+}}{R-\tau_{+}}\right\}^{1 / 2}-\frac{1}{\tan \left(\theta_{\mathrm{B}}-\chi\right)},
$$

and for the expansion side of the crystal,

$$
\Delta \phi_{-}=\left\{\frac{1}{\left[\tan \left(\theta_{\mathrm{B}}-\chi\right)\right]^{2}}+\frac{2 \tau_{-}}{R+\tau_{-}}\right\}^{1 / 2}-\frac{1}{\tan \left(\theta_{\mathrm{B}}-\chi\right)} .
$$

The total change in $\phi$ across the entire bent crystal is then

$$
\begin{aligned}
\Delta \phi= & \Delta \phi_{+}-\Delta \phi_{-} \\
= & \left\{\frac{1}{\left[\tan \left(\theta_{\mathrm{B}}-\chi\right)\right]^{2}}-\frac{2 \tau_{+}}{R-\tau_{+}}\right\}^{1 / 2} \\
& -\left\{\frac{1}{\left[\tan \left(\theta_{\mathrm{B}}-\chi\right)\right]^{2}}-\frac{2 \tau_{-}}{R-\tau_{-}}\right\}^{1 / 2} \\
\simeq & -\frac{\tau_{+}+\tau_{-}}{R} \tan \left(\theta_{\mathrm{B}}-\chi\right) \\
= & -\frac{T}{R} \tan \left(\theta_{\mathrm{B}}-\chi\right) .
\end{aligned}
$$

Note that the derivation for $\Delta \phi$ is based on the assumption that the source distance is infinite. A subscript $\infty$ will be used for clarity:

$$
\Delta \phi_{\infty}=-\frac{T}{R} \tan \left(\theta_{\mathrm{B}}-\chi\right),
$$

where $\infty$ stands for the infinite source distance scenario. The general solution for $\Delta \phi$ will be given in Appendix B3.

\section{APPENDIX $B$}

Feature angles in general bent Laue diffraction with a finite source distance

With a finite source distance, the relations between the feature angles in bent Laue diffraction are shown in Fig. 14. $\left(\theta_{\mathrm{B}_{0}}\right.$ is equivalent to $\theta_{\mathrm{B}}$ and $\chi_{0}$ is equivalent to $\chi$ where applicable.)

B1. $\psi$

From the geometry shown in Fig. 4, we can easily get

$$
\overline{A B} \cos \left(\theta_{\mathrm{B}_{0}}-\chi_{0}\right)=T,
$$

$$
\overline{A B} \sin 2 \theta_{\mathrm{B}_{0}}=h,
$$

which gives

$$
h=\frac{T \sin 2 \theta_{\mathrm{B}_{0}}}{\cos \left(\chi-\theta_{\mathrm{B}_{0}}\right)} .
$$

The X-ray incident-angle divergence between points $A$ and $B$ is given by

$$
\psi=\frac{h}{p}=\frac{T}{p} \frac{\sin 2 \theta_{\mathrm{B}_{0}}}{\cos \left(\chi-\theta_{\mathrm{B}_{0}}\right)} .
$$

\section{B2. $\Theta$}

$\Theta$ represents the angular distance from $A A^{\prime}$ to $B B^{\prime}$ and is defined with counterclockwise as positive. An important relationship between the X-rays diffracted from points $A$ and $B$ is that

$$
2 \theta_{\mathrm{B}_{0}}+\Theta=2 \theta_{\mathrm{B}_{1}}+\psi,
$$

or

$$
\begin{aligned}
\Theta & =2 \theta_{\mathrm{B}_{1}}-2 \theta_{\mathrm{B}_{0}}+\psi \\
& =T\left[\frac{1+v}{R} \sin 2 \chi-\frac{2}{R} \tan \left(\theta_{\mathrm{B}}-\chi\right)-\frac{1}{p} \frac{\sin 2 \theta_{\mathrm{B}}}{\cos \left(\chi-\theta_{\mathrm{B}}\right)}\right],
\end{aligned}
$$

which can be easily observed in Fig. 14 .

When the magic condition is met, $A A^{\prime}$ and $B B^{\prime}$ overlap and $\Theta=0$, which indicates that the magic condition with a finite source distance is given by

$$
2 \theta_{\mathrm{B}_{0}}-2 \theta_{\mathrm{B}_{1}}-\psi=0,
$$

or

$$
\Delta \phi-\Delta \chi=-\frac{1}{2} \psi .
$$

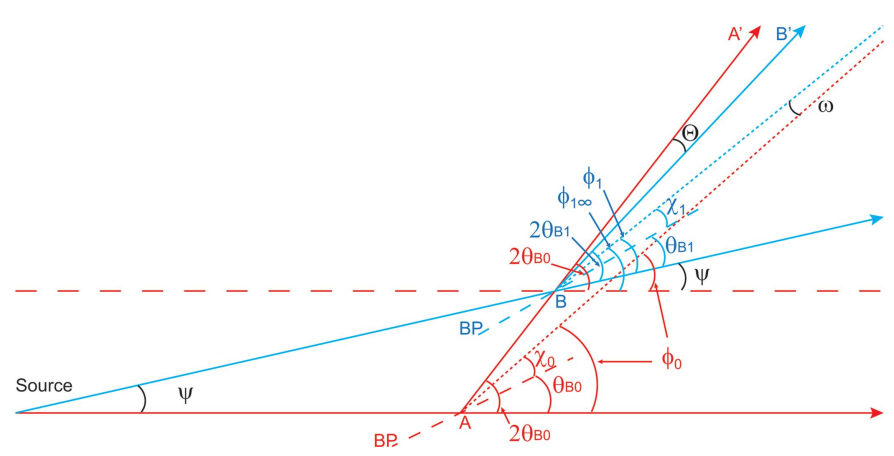

Figure 14

Feature angles in general bent Laue diffraction with a finite source distance. Point $A$ is the location where the reference X-ray enters the crystal and gets diffracted, and point $B$ is where the diffracted reference $\mathrm{X}$-ray $\left(A A^{\prime}\right)$ exits the crystal. 'BP' stands for Bragg plane. The rays are organized by colour for two rays coming from the source. 
B3. $\Delta \phi$

As shown in Fig. 14,

$$
\phi_{1}=\phi_{1 \infty}-\psi
$$

Therefore, the change in $\phi$ angle for a finite source distance is given by

$$
\begin{aligned}
\Delta \phi & =\Delta \phi_{\infty}-\psi \\
& =-\frac{T}{R} \tan \left(\theta_{\mathrm{B}}-\chi\right)-\frac{T}{p} \frac{\sin 2 \theta_{B}}{\cos \left(\chi-\theta_{\mathrm{B}}\right)} .
\end{aligned}
$$

Combined with equation (93), the magic condition with a finite source distance can be described by

$$
\Delta \phi_{\infty}-\Delta \chi-\frac{\psi}{2}=0
$$

or

$$
\frac{1+v}{2} \frac{T}{R} \sin 2 \chi-\frac{T}{R} \tan \left(\theta_{\mathrm{B}}-\chi\right)-\frac{1}{2} \frac{T}{p} \frac{\sin 2 \theta_{\mathrm{B}}}{\cos \left(\chi-\theta_{\mathrm{B}}\right)}=0 .
$$

B4. $\Delta \theta_{\mathrm{B}_{01}}$

The difference between the Bragg angles at points $A$ and $B$ for general bent Laue diffraction is

$$
\begin{aligned}
\Delta \theta_{\mathrm{B}_{01}} & =\theta_{\mathrm{B}_{1}}-\theta_{\mathrm{B}_{0}} \\
& =\Delta \phi-\Delta \chi \\
& =\frac{1+v}{2} \frac{T}{R} \sin 2 \chi-\frac{T}{R} \tan \left(\theta_{\mathrm{B}}-\chi\right)-\frac{T}{p} \frac{\sin 2 \theta_{\mathrm{B}}}{\cos \left(\chi-\theta_{\mathrm{B}}\right)} .
\end{aligned}
$$

Equation (98) is also equivalent to

$$
\Delta \theta_{\mathrm{B}_{01}}=\Delta \theta_{\mathrm{B}_{\mathrm{mis}}}-\frac{\psi}{2} .
$$

When the magic condition is met, $\Delta \theta_{\mathrm{B}_{\mathrm{mis}}}=0$ and

$$
\Delta \theta_{\mathrm{B}_{01}}^{\mathrm{mc}}=-\frac{\psi}{2}=-\frac{1}{2} \frac{T}{p} \frac{\sin 2 \theta_{\mathrm{B}}}{\cos \left(\chi-\theta_{\mathrm{B}}\right)},
$$

where the superscript ' $m c$ ' stands for the 'magic condition' scenario.

\section{B5. $\chi_{1}$ and $\chi_{2}$}

$\chi_{1}$ and $\chi_{2}$ are the asymmetry angles of the Bragg planes at points $B$ and $C$, respectively. The change in asymmetry angle is caused only by the bending deformation of the crystal, and thus for locations at the same depth inside the crystal the asymmetry angles are the same. The change in $\chi$ over the crystal is given by equation (3). $\chi_{1}$ and $\chi_{2}$ are then given by

$$
\chi_{2} \equiv \chi_{1}=\chi_{0}+\Delta \chi
$$

B6. $\theta_{\mathrm{B}_{1}}$

As shown in Fig. 9, $\theta_{\mathrm{B}_{1}}$ is the Bragg diffraction angle at point $B$ where the reference diffraction ray, $A A^{\prime}$, exits the crystal.
When the magic condition is met, $\theta_{\mathrm{B}_{1}}^{\mathrm{mc}}$ is simply given by

$$
\begin{aligned}
\theta_{\mathrm{B}_{1}}^{\mathrm{mc}} & =\theta_{\mathrm{B}_{0}}+\Delta \theta_{\mathrm{B}_{01}}^{\mathrm{mc}} \\
& =\theta_{\mathrm{B}_{0}}-\psi / 2 .
\end{aligned}
$$

When the magic condition is not met, $\theta_{\mathrm{B}_{1}}$ is given by

$$
\begin{aligned}
\theta_{\mathrm{B}_{1}}= & \theta_{\mathrm{B}_{0}}+\Delta \theta_{\mathrm{B}_{01}} \\
= & \theta_{\mathrm{B}_{0}}+\Delta \phi-\Delta \chi \\
= & \theta_{\mathrm{B}_{0}}+\Delta \phi_{\infty}-\Delta \chi-\psi \\
= & \theta_{\mathrm{B}_{0}}-\frac{T}{R} \tan \left(\theta_{\mathrm{B}_{0}}-\chi_{0}\right)+\frac{1+v}{2} \frac{T}{R} \sin 2 \chi_{0} \\
& -\frac{T \sin 2 \theta_{\mathrm{B}_{0}}}{p \cos \left(\chi_{0}-\theta_{\mathrm{B}_{0}}\right)} .
\end{aligned}
$$

B7. $\theta_{\mathrm{B}_{2}}$

As shown in Fig. 9, $\theta_{\mathrm{B}_{2}}$ is the Bragg diffraction angle at point $C$ where the edge ray, $C C^{\prime}$, of the quasi-mono beam is diffracted on the downstream surface of the crystal. The Bragg angle, $\theta_{\mathrm{B}_{2}}$, at point $C$ can be described with the Bragg angle deviation, $\Delta \theta_{\mathrm{B}_{12}}$ from point $B$ to point $C$. $\Delta \theta_{\mathrm{B}_{12}}$ can be described using the quasi-mono-beam footprint, $L$, on the downstream side of the crystal, i.e. the rotation of the Bragg planes between points $B$ and $C$ and the rotation of the incident rays at points $B$ and $C$ relative to the source. Thus, $\theta_{\mathrm{B}_{2}}$ can be described by

$$
\begin{aligned}
\theta_{\mathrm{B}_{2}} & =\theta_{\mathrm{B}_{1}}+\Delta \theta_{\mathrm{B}_{12}} \\
& =\theta_{\mathrm{B}_{1}}-\frac{L}{R}-\frac{L \cos \left(\chi_{2}+\theta_{\mathrm{B}_{2}}\right)}{p} \\
& \simeq \theta_{\mathrm{B}_{1}}-\frac{L}{R}-\frac{L \cos \left(\chi_{0}+\theta_{\mathrm{B}_{0}}\right)}{p} .
\end{aligned}
$$

Note that although $\theta_{\mathrm{B}_{2}}$ is present in equation (34) for the calculation of $L$, by approximating $L$ to the upstream surface of the crystal, more accurate values for $L$ and $\theta_{\mathrm{B}_{2}}$ can be approached recursively.

\section{APPENDIX C}

Supplemental materials to magic condition energy resolution

\section{C1. Derivation of $(\Delta E / E)_{1}$}

As shown in Fig. 15, we can easily get the relations

$$
\begin{gathered}
\frac{h_{1}}{R-T}=\frac{h_{0}}{R}, \\
\frac{d_{1} / \cos \chi_{1}}{R-T}=\frac{d_{0} / \cos \chi_{0}}{R}, \\
d_{1}=\left(1-\frac{T}{R}\right) \frac{\cos \chi_{1}}{\cos \chi_{0}} d_{0},
\end{gathered}
$$

where 


$$
\begin{aligned}
\cos \chi_{1} & =\cos \left(\chi_{0}+\Delta \chi\right) \\
& =\cos \chi_{0} \cos \Delta \chi-\sin \chi_{0} \sin \Delta \chi \\
& \simeq \cos \chi_{0}-\sin \chi_{0} \Delta \chi .
\end{aligned}
$$

Equation (116) gives the first-order small-angle approximation on $\Delta \chi$.

Combining equations (113) and (116), we have

$$
\begin{aligned}
d_{1} & =\left(1-\frac{T}{R}\right) \frac{d_{0}}{\cos \chi_{0}}\left(\cos \chi_{0}-\sin \chi_{0} \Delta \chi\right) \\
& =d_{0}\left(1-\frac{T}{R}\right)\left(1-\tan \chi_{0} \Delta \chi\right) .
\end{aligned}
$$

$\Delta \chi$ is given by equation (3), and we recall it here:

$$
\Delta \chi=-\frac{1+v}{2} \frac{T}{R} \sin 2 \chi_{0}
$$

Therefore,

$$
\begin{aligned}
\frac{\Delta d}{d}= & \frac{d_{1}-d_{0}}{d_{0}} \\
= & \frac{T}{R} \Delta \chi \tan \chi_{0}-\Delta \chi \tan \chi_{0}-\frac{T}{R} \\
= & -\frac{1+v}{2}\left(\frac{T}{R}\right)^{2} \sin 2 \chi_{0} \tan \chi_{0} \\
& +\frac{1+v}{2} \frac{T}{R} \sin 2 \chi_{0} \tan \chi_{0}-\frac{T}{R} \\
\simeq & \frac{1+v}{2} \frac{T}{R} \sin 2 \chi_{0} \tan \chi_{0}-\frac{T}{R} \quad\left(\frac{T}{R}\right)^{2} \simeq 0 \\
= & \frac{1+v}{2} \frac{T}{R} 2 \sin ^{2} \chi_{0} \cos \chi_{0} \frac{\sin \chi_{0}}{\cos \chi_{0}}-\frac{T}{R} \\
= & (1+v) \frac{T}{R} \sin ^{2} \chi_{0}-\frac{T}{R} \\
= & \frac{T}{R}\left[(1+v) \sin ^{2} \chi_{0}-1\right] \\
= & \frac{T}{R}\left(v \sin ^{2} \chi_{0}-\cos ^{2} \chi_{0}\right) .
\end{aligned}
$$

Thus, the contribution to the energy resolution by latticespacing variation is

$$
\left(\frac{\Delta E}{E}\right)_{1}=-\frac{\Delta d}{d}=\frac{T}{R}\left(\cos ^{2} \chi_{0}-v \sin ^{2} \chi_{0}\right) .
$$

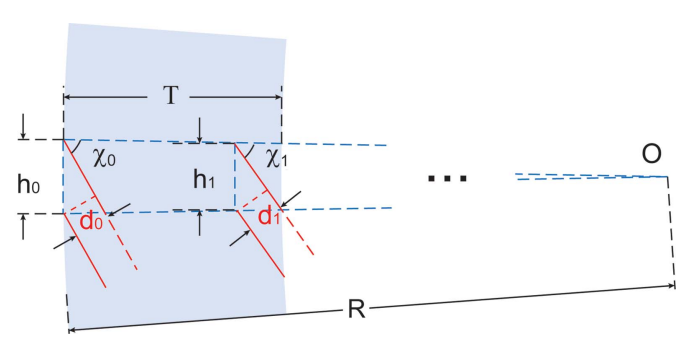

Figure 15

The lattice-spacing variation caused by the crystal deformation

\section{C2. Derivation of $(\Delta E / E)_{2}$}

When the source distance is finite, the X-rays that arrive at points $A$ and $B$ form an angle $\psi$ or $\psi_{A B}$ (Fig. 9), which is given by equation $(8)$, and we recall it here:

$$
\psi=\frac{T}{p} \frac{\sin 2 \theta_{\mathrm{B}}}{\cos \left(\chi-\theta_{\mathrm{B}}\right)} .
$$

As a consequence, to meet the magic condition, the effective Bragg angles at points $A$ and $B$ vary by $\Delta \theta_{\mathrm{B}_{01}}$, which is given by equation (7), and we recall it here:

$$
\Delta \theta_{\mathrm{B}_{01}}=-\frac{1}{2} \psi
$$

Therefore, the energy spread caused by a finite source-tocrystal distance can be described by

$$
\begin{aligned}
\left(\frac{\Delta E}{E}\right)_{2} & =-\frac{\Delta \theta_{\mathrm{B}_{01}}}{\tan \theta_{\mathrm{B}}} \\
& =\frac{\psi}{2 \tan \theta_{\mathrm{B}}} \\
& =\frac{T \cos ^{2} \theta_{\mathrm{B}}}{p \cos \left(\chi-\theta_{\mathrm{B}}\right)} .
\end{aligned}
$$

C3. Integration of energy resolution contributions from $(\Delta E / E)_{1}$ and $(\Delta E / E)_{2}$

While the contributions from the Darwin width, source size or detector spatial resolution are independent of other contributions, the contributions originating from the $d$ spacing and source distance can either supplement or counteract each other and their values should be treated with signs. The integration of these two contributions in different scenarios is explained below.

(i) With a finite source distance and ignoring the latticespacing variation in the crystal, the Bragg angle at point $B$ is smaller than that at point $A\left(\theta_{\mathrm{B}_{1}}<\theta_{\mathrm{B}_{0}}\right)$ and the energy of the Bragg-diffracted $\mathrm{X}$-rays at point $B$ is higher than that at point $A\left(E_{1}>E_{0}\right)$ because of Bragg's law.

When the lattice-spacing variation is considered, in a focusing (defocusing) geometry, it supplements (counteracts) the energy deviation caused by the source distance, because the lattice spacing is compressed (expanded) on the downstream side of the crystal, which corresponds to a higher (lower) energy of the Bragg-diffracted X-rays.

(ii) With a beyond-infinite source distance (a virtual source or converging incident beam or negative source distance), the Bragg angle at point $B$ is larger than that at point $A\left(\theta_{\mathrm{B}_{1}}>\theta_{\mathrm{B}_{0}}\right)$ and the energy of the Bragg-diffracted X-rays at point $B$ is lower than that at point $A\left(E_{1}<E_{0}\right)$ because of Bragg's law.

When the lattice-spacing variation is considered, in a focusing (defocusing) geometry, it counteracts (supplements) the energy deviation caused by the source distance, because the lattice spacing is compressed (expanded) on the downstream side of the crystal, which corresponds to a higher (lower) energy of the Bragg-diffracted X-rays. 


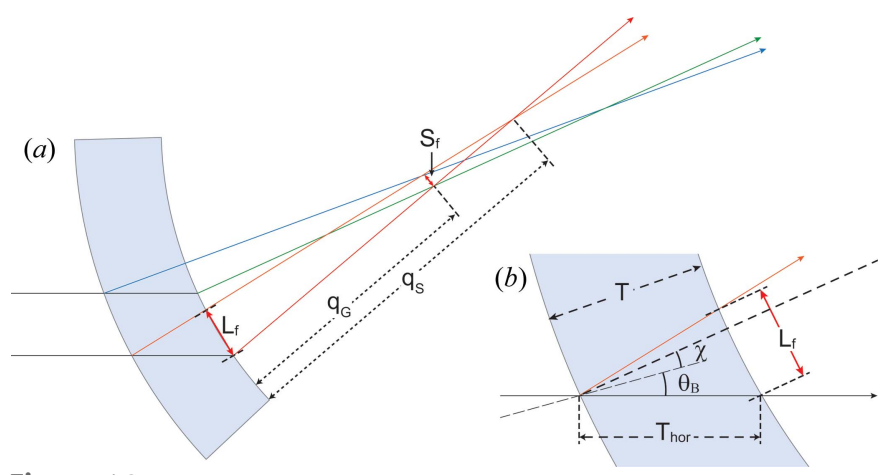

Figure 16

(a) The focal size in relation to the single-ray focus and the geometric focus. (b) An expanded view to show the angles associated with the bottom rays in $(a)$.

\section{APPENDIX $D$}

\section{Focal size calculation with two types of focus}

The focal size $S_{\mathrm{f}}$ of a bent Laue crystal for a point source can be calculated using the geometry of the single-ray focus and geometric focus. As shown in Fig. 16, the focal size is given by

$$
S_{\mathrm{f}}=\frac{q_{\mathrm{S}}-q_{\mathrm{G}}}{q_{\mathrm{S}}} L_{\mathrm{f}}
$$

where $L_{\mathrm{f}}$ is the length of the crystal illuminated by a single-ray focused fan beam and is given by

$$
\begin{aligned}
L_{\mathrm{f}} & =T_{\text {hor }} \sin 2 \theta_{\mathrm{B}} \\
& =\frac{\sin 2 \theta_{\mathrm{B}}}{\cos \left(\chi+\theta_{\mathrm{B}}\right)} T .
\end{aligned}
$$

$q_{\mathrm{S}}$ and $q_{\mathrm{G}}$ are the single-ray focal distance and the geometric focus and are given by equations (17) and (16), respectively.

\section{APPENDIX $E$}

The energy spread of the quasi-mono beam caused by a finite source distance, $(\Delta E / E)_{\mathrm{Q} 2}$

$(\Delta E / E)_{\mathrm{Q} 2}$ or the energy spread of the quasi-mono beam caused by a finite source distance can be described as the difference between the incident X-ray Bragg angles at points $A$ and $C\left(\Delta \theta_{\mathrm{Q} 2}\right)$.

As shown in Fig. 9, with a point source, the X-rays hitting points $A$ and $C$ form a pitch angle $\psi_{A C}$ at the source. Note that the subscripts of the $\psi$ angles indicate the direction of the angle. For example, $\psi_{A C}$ represents the angle from $E A$ to $E C$ (counterclockwise as positive). As a result, to ensure the output beam is parallel, the difference between the Bragg angles at points $A$ and $C$ is

$$
\Delta \theta_{\mathrm{Q} 2}=\theta_{\mathrm{B}_{2}}-\theta_{\mathrm{B}_{0}}=-\frac{1}{2} \psi_{A C},
$$

where $\psi_{A C}$ can be described by

$$
\psi_{A C}=\psi_{A B}+\psi_{B C} .
$$

$\psi_{A B}$ is given by equation (8), and we recall it here:

$$
\psi_{A B}=\frac{T}{p} \frac{\sin 2 \theta_{\mathrm{B}_{0}}}{\cos \left(\chi_{0}-\theta_{\mathrm{B}_{0}}\right)}
$$

$\psi_{B C}$ can be calculated from the length of the quasi-monobeam footprint, $L$, and the source distance, $p$. The direction of the incident $\mathrm{X}$-rays at point $B$ is away from the crystal surface normal by $\left(\theta_{\mathrm{B}_{2}}+\chi_{2}\right)$. Thus,

$$
\begin{aligned}
\psi_{B C} & =\frac{L \cos \left(\chi_{2}+\theta_{\mathrm{B}_{2}}\right)}{p} \\
& \simeq \frac{L \cos \left(\chi_{0}+\theta_{\mathrm{B}_{0}}\right)}{p} .
\end{aligned}
$$

Therefore,

$$
\begin{aligned}
\Delta \theta_{\mathrm{Q} 2} & =-\frac{1}{2} \psi_{A C} \\
& =-\frac{1}{2}\left(\psi_{A B}+\psi_{B C}\right) \\
& =-\frac{1}{2}\left[\frac{T \sin 2 \theta_{\mathrm{B}_{0}}}{p \cos \left(\chi_{0}-\theta_{\mathrm{B}_{0}}\right)}+\frac{L \cos \left(\chi_{2}+\theta_{\mathrm{B}_{2}}\right)}{p}\right] \\
& \simeq-\frac{1}{2}\left[\frac{T \sin 2 \theta_{\mathrm{B}_{0}}}{p \cos \left(\chi_{0}-\theta_{\mathrm{B}_{0}}\right)}+\frac{L \cos \left(\chi_{0}+\theta_{\mathrm{B}_{0}}\right)}{p}\right] .
\end{aligned}
$$

Using Bragg's law,

$$
\begin{aligned}
\left(\frac{\Delta E}{E}\right)_{\mathrm{Q} 2} & =-\frac{\Delta \theta_{\mathrm{Q}_{2}}}{\tan \theta_{\mathrm{B}_{0}}} \\
& =\frac{1}{2 \tan \theta_{\mathrm{B}_{0}}}\left[\frac{T \sin 2 \theta_{\mathrm{B}_{0}}}{p \cos \left(\chi_{0}-\theta_{\mathrm{B}_{0}}\right)}+\frac{L \cos \left(\chi_{2}+\theta_{\mathrm{B}_{2}}\right)}{p}\right] \\
& \simeq \frac{1}{2 \tan \theta_{\mathrm{B}_{0}}}\left[\frac{T \sin 2 \theta_{\mathrm{B}_{0}}}{p \cos \left(\chi_{0}-\theta_{\mathrm{B}_{0}}\right)}+\frac{L \cos \left(\chi_{0}+\theta_{\mathrm{B}_{0}}\right)}{p}\right] .
\end{aligned}
$$

\section{APPENDIX $\boldsymbol{F}$}

\section{Summary of symbols}

Variables are defined when they are used for the first time in the text, and some important variable definitions are summarized in this appendix for convenience.

\section{F1. Source distance, $p$}

$p$ is positive when there is a real source or the incident beam to the crystal is diverging.

\section{F2. Crystal bending radius, $R$}

$R$ is positive when the $\mathrm{X}$-ray beam is incident on the convex side of the crystal. The surface curvature of a crystal bent with a positive $R$ focuses the incident beam. 
Table 1

List of parameter signs for a real focus (+) or virtual focus (-) due to the curvature of the diffraction planes.

\begin{tabular}{llll}
\hline$R$ & $\theta_{\mathrm{B}}$ & $\chi$ & Type of focus \\
\hline+ & + & + & + \\
+ & + & - & - \\
+ & - & + & - \\
+ & - & - & + \\
- & + & + & - \\
- & + & - & + \\
- & - & + & + \\
- & - & - & - \\
\hline
\end{tabular}

\section{F3. Crystal thickness, $T_{0}$ and $T$}

$T_{0}$ is the thickness of the crystal in the absence of bending, and $T$ is the thickness of the bent crystal. $T_{0}$ and $T$ are coordinate values relative to the neutral reference plane rather than absolute values, which might be expected for a thickness. $T_{0}$ and $T$ can be positive or negative. When they are negative, $T_{0}$ and $T$ are on the upstream side of the reference plane.

\section{F4. Bragg angle, $\theta_{\mathrm{B}}$}

The central incident-ray Bragg angle on the crystal reference surface. It is directed from the X-ray incident direction to the diffraction plane.

When the direction is counterclockwise, $\theta_{\mathrm{B}}$ is positive and the $\mathrm{X}$-ray is bounced up; when the direction is clockwise, $\theta_{\mathrm{B}}$ is negative and the X-ray is bounced down.

\section{F5. Asymmetry angle, $\chi$}

The directed angle from the diffraction plane to the crystal surface normal. When the direction is counterclockwise, $\chi$ is positive; when the direction is clockwise, $\chi$ is negative.

$\chi$ influences the consistency of the focusing properties of the diffraction planes and the crystal surface geometry. As an example, in the case of an up bounce $(\theta>0)$ and focusing crystal geometry $(R>0)$, a positive $\chi$ angle corresponds to diffraction-plane focusing and a negative $\chi$ angle corresponds to diffraction-plane defocusing. The influences of all $R, \theta_{\mathrm{B}}$ and $\chi$ are listed in Table 1 (a positive sign for the diffraction planes represents real focus and a negative sign represents virtual focus). An intuitive way of understanding this is that the sign of the product of the three variables corresponds to focusing or defocusing by the diffraction planes.

\section{F6. Poisson ratio, $v$}

The Poisson ratio is considered as homogeneous with a given crystal material and diffraction indices.

F7. $\Delta \chi$

$$
\Delta \chi=\chi_{1}-\chi_{0}
$$

where $\chi_{1}$ is the asymmetry angle of the diffraction planes at point $B$ and is equal to the asymmetry angle at any point on the same crystal surface; $\chi_{0}$ is the asymmetry angle of the diffraction planes at point $A$ and equals the asymmetry angle at any point on the same crystal surface.

F8. $\Delta \phi$ and $\Delta \phi_{\infty}$

$$
\Delta \phi=\phi_{1}-\phi_{0},
$$

where $\phi$ stands for the directed angle from the crystal surface normal to the diffracted X-rays, and the subscripts 1 and 0 correspond to points $B$ and $A$, respectively. The angle $\phi$ is equivalent to $\theta_{\mathrm{B}}-\chi$ and is used for the convenience of concept illustration.

$\Delta \phi_{\infty}$ is the $\Delta \phi$ given an infinite source distance. The relationship between the general $\Delta \phi$ and $\Delta \phi_{\infty}$ is given by

$$
\Delta \phi=\Delta \phi_{\infty}-\psi
$$

where $\psi$ is the opening angle between X-rays incident at points $A$ and $B$ (Fig. 14).

\section{F9. Quasi-monochromatic-beam width, W}

The width of the diffracted beam parallel to the diffracted reference X-ray beam. The sign of the width value corresponds to the relative direction of the quasi-mono beam to the diffracted reference X-ray beam.

F10. Quasi-mono-beam footprint length, $L$

The quasi-monochromatic-beam footprint arc length on the downstream surface of the crystal.

\section{Funding information}

This research was funded by the Canadian Network for Research and Innovation in Machining Technology, Natural Sciences and Engineering Research Council of Canada (grant to DC, PQ and NS). Part or all of the research described in this paper was performed at the Canadian Light Source, a national research facility of the University of Saskatchewan, which is supported by the Canada Foundation for Innovation (CFI), the Natural Sciences and Engineering Research Council (NSERC), the National Research Council (NRC), the Canadian Institutes of Health Research (CIHR), the Government of Saskatchewan and the University of Saskatchewan.

\section{References}

Bassey, B., Martinson, M., Samadi, N., Belev, G., Karanfil, C., Qi, P. \& Chapman, D. (2016). Phys. Med. Biol. 61, 8180-8198.

Cauchois, Y. (1932). J. Phys. Radium, 3, 320-336.

Martinson, M., Samadi, N., Bassey, B., Gomez, A. \& Chapman, D. (2015). J. Synchrotron Rad. 22, 801-806.

Penning, P. \& Polder, D. (1961). Philips Res. Rep, 16, 419-440.

Qi, P., Samadi, N., Martinson, M., Ponomarenko, O., Bassey, B., Gomez, A., George, G. N., Pickering, I. J. \& Chapman, L. D. (2019). Sci. Rep. 9, 17734.

Qi, P., Shi, X., Samadi, N. \& Chapman, D. (2019). Proc. SPIE, 11108E, 12.

Schulze, C. \& Lienert, U. (1996). ESRF Newslett. 25, 38-40.

Schulze, C., Lienert, U., Hanfland, M., Lorenzen, M. \& Zontone, F. (1998). J. Synchrotron Rad. 5, 77-81. 


\section{research papers}

Suortti, P., Lienert, U. \& Schulze, C. (1994). Nucl. Instrum. Methods Phys. Res. A, 338, 27-32.

Takagi, S. (1969). J. Phys. Soc. Jpn, 26, 1239-1253.

Taupin, D. (1964). Bull. Soc. Fr. Minéral. Cristallogr. 87, 469-511.

Tolentino, H., Dartyge, E., Fontaine, A. \& Tourillon, G. (1988). J. Appl. Cryst. 21, 15-22.
Tolentino, H., Dartyge, E., Fontaine, A. \& Tourillon, G. (1989). Physica B, 158, 317-321.

Zachariasen, W. H. (1945). Theory of X-ray Diffraction in Crystals. New York: John Wiley \& Sons.

Zhu, Y., Samadi, N., Martinson, M., Bassey, B., Wei, Z., Belev, G. \& Chapman, D. (2014). Phys. Med. Biol. 59, 2485-2503. 